\title{
ASYMPTOTIC EXPANSION OF SOLUTIONS TO THE NONLINEAR SCHRÖDINGER EQUATION WITH POWER NONLINEARITY
}

\author{
Satoshi MASAKI \\ (Received 26 June 2007)
}

\begin{abstract}
We study an asymptotic expansion near $t=\infty$ of the solution to the Cauchy problem for the nonlinear Schrödinger equation with repulsive short-range nonlinearity of power type. We construct two kinds of approximate solution with asymptotic expansions. The first is an accurate approximate solution and of abstract form. The second is the approximation of the first and of explicit form. The sharpness of these approximations strongly depend on the fractional part of the power of the nonlinearity. In particular, if the power is an integer, we obtain a complete expansion of the solution.
\end{abstract}

\section{Introduction}

This paper is devoted to the study of the asymptotic behavior of the solution to the Cauchy problem of the nonlinear Schrödinger equation:

$$
i \partial_{t} u+\Delta u=f(u), \quad u(0, x)=\phi,
$$

where $u$ is a complex valued unknown function of $(t, x) \in \mathbb{R}^{1+N}, \partial_{t}=\partial / \partial t, \Delta$ is the Laplacian in $\mathbb{R}^{N}$, and $f$ denotes a nonlinear interaction given by a complex-valued function $f$ on $\mathbb{C}$. In this paper, we assume that $f$ is a single-power nonlinearity satisfying the Gauge invariance and repulsive conditions. Namely we assume that $f$ is of the form

$$
f(u)=|u|^{\alpha} u
$$

with a positive number $\alpha$.

There is a large amount of literature on the Cauchy problem and the scattering theory for (1.1) (see, for instance, [2-13, 15-17], and references therein). The usual scattering theory for (1.1) compares the full dynamics given by the solutions to (1.1) and the free dynamics described by the free propagator $U(t)=\exp i t \Delta$. In the case of repulsive interactions, the existence and asymptotic completeness of wave operators for (1.1) with (1.2) have been proved in the space $X_{1,1}=H^{1} \cap \mathcal{F}\left(H^{1}\right)$ for any $\alpha$ with $\gamma(N) \leq \alpha<\alpha_{0}(N)$, where $H^{1}$ is the Sobolev space of order one on $\mathbb{R}^{N}$ defined by $H^{1}=(1-\Delta)^{-1 / 2} L^{2}\left(\mathbb{R}^{N}\right), \mathcal{F}$ denotes the Fourier transform, $\gamma(N)=\left(2-N+\sqrt{N^{2}+12 N+4}\right) /(2 N)$, and $\alpha_{0}(N)=4 /(N-2)$ if $N \geq 3$ and $\alpha_{0}(N)=\infty$ if $N \leq 2$. From the view point of the asymptotic behavior of the

2000 Mathematics Subject Classification: Primary 35Q55, 35C20, 35A35.

Keywords: nonlinear Schrödinger equation; asymptotic behavior of the solution; asymptotic expansion.

(c) 2009 Faculty of Mathematics, Kyushu University 
solution $u$, the scattering theory means that the nonlinear effect becomes small near $t= \pm \infty$, and so that the solution asymptotically behaves as a free solution. In other words, if we rewrite (1.1) as

$$
u(t)=U(t) \phi_{+}+i \int_{t}^{\infty} U(t-s) f(u(s)) d s
$$

with the scattered state for positive time $\phi_{+}=\lim _{t \rightarrow \infty} U(-t) u(t) \in X_{1,1}$, then the scattering theory implies that the latter term converges to zero as $t \rightarrow \infty$. It can be said that $U(t) \phi_{+}$is the first approximate solution of $u$ in large time. The second approximate solution to (1.1) with (1.2) for scattering is given in [12] for the case $1 \leq N \leq 5$ and $\gamma(N)<\alpha<\alpha_{0}(N)$ under a suitable regularity condition on the data. The second approximation term was first constructed in [13] under several restrictive assumptions except for the case of integrable systems (see also [11]). For (1.1) with the Hartree-type nonlinearity, the second and higher approximate solutions are understood in the long-range as well as in the short-range case (see [18]).

In this paper, we give two sequences of functions under the assumption that $\alpha \in$ $\left(\gamma(N), \alpha_{0}(N)\right)$. We restrict our attention to positive time. We note beforehand that the results in this paper also hold for negative time. The first is the sequence $\left\{w_{n}(t)\right\}_{n=0}$. The function $w_{0}$ is the free solution $e^{i t \Delta} \phi_{+}$. The $(n+1)$ th approximate solution $w_{n}$, which is defined by a recursive formula from $w_{0}$, approximates $u-\sum_{k=0}^{n-1} w_{k}$ for all $n \geq 1$, and therefore the sums $\sum_{k=0}^{n} w_{k}$ yield the approximation of the solution for (1.1) with (1.2) near $t=\infty$. Although the sums of $w_{n}$ are good approximate solutions, these functions $w_{n}$ themselves take very complicated abstract form. Then, under the suitable regularity of the initial data, we construct the functions of the phase-amplitude form, denoted by $V_{n}$, which approximate the previous $w_{n}$ near $t=\infty$. This approximation of $w_{n}$ not only clarifies the asymptotic form of $w_{n}$ as $t \rightarrow \infty$, but also gives us the lower estimates of $w_{n}$ and $u-\sum_{k=0}^{n} w_{k}$.

The accuracy of approximation of $u$ by $\sum_{k=0}^{n} w_{k}$ strongly depends on the fractional part of the power $\alpha$. The upper bound of the accuracy goes to infinity as $\alpha$ tends to an integer from below. In particular, if the power is an integer, then the approximation can be made accurate arbitrarily. Furthermore, it gives a complete expansion of the solution $u$, that is, the infinite sum $\sum_{k=0}^{\infty} w_{k}$ converges to the true solution in strong topology (see Theorem 1). In the special case that $\alpha$ is an even number, we have slightly different constructions of $w_{n}$ which are based on the property that $|u|^{\alpha} u$ can be written as $u^{a} \bar{u}^{b}$ with $a, b \in \mathbb{N}$. The sum $\sum_{k=0}^{n} w_{k}$ converges to the solution in a topology stronger than that in the previous case. In particular, it does so in $L^{r}\left(\mathbb{R}^{N}\right)$ for all $r \in[2, \infty]$.

The approximation of $w_{n}$ by $V_{n}$ is possible only for $n=0,1$ in general. However, in the special case when $\alpha$ is an even number, we can construct $V_{n}$ for all $n \geq 0$ and so the lower bound estimates of $w_{n}$ and $u-\sum_{k=0}^{n} w_{k}$ hold for all $n$ (see Theorem 4).

In [12], the authors have used a second approximate solution of $u$, that is, an approximation of $u-w_{0}$. For our notation, the function $V_{1}$ (the approximation of $w_{1}$ ) corresponds to it. Our result is better in the following two respects. First, we also show the same asymptotics for $N \leq 8$ (see Theorem 3 and Remark 1.7). Second, we obtain several ways to construct a more accurate approximate solution. If $n$ is large then the sum $\sum_{k=1}^{n} w_{k}$ gives us better approximation of $u-w_{0}$ in some cases with $X_{1,1}$ data (see Remark 1.3). Moreover, under the suitable regularity, $w_{1}$ alone provides a better approximation (see Theorem 2). 
To state our main result precisely, we introduce the following notation. For any $r$ with $1 \leq r \leq \infty, L^{r}=L^{r}\left(\mathbb{R}^{N}\right)$ denotes the Lebesgue space on $\mathbb{R}^{N}$. For any $s \in \mathbb{R}$ and any $r$ with $1<r<\infty, H_{r}^{s}=(1-\Delta)^{-s / 2} L^{r}$ and $\dot{H}_{r}^{s}=(-\Delta)^{-s / 2} L^{r}$ denote the Sobolev space defined in terms of Bessel potentials and the homogeneous Sobolev space defined in terms of Riesz potentials, respectively. We write $H^{s}=H_{2}^{s}$ and $\dot{H}^{s}=\dot{H}_{2}^{s}$, for simplicity. For any $s \in \mathbb{R}$ and any $r$ with $1 \leq r \leq \infty, \dot{B}_{r}^{s}=\dot{B}_{r, 2}^{s}$ denotes the homogeneous Besov space. For $a, b \in \mathbb{R}$ we denote by $a \vee b$ and $a \wedge b$ the maximum and minimum, respectively. For the free propagator $U(t)=\exp (i t \Delta)$ we use the factorization for $t \neq 0: U(t)=M(t) D(t) \mathcal{F} M(t)$, where $M(t)=\exp \left(i|x|^{2} /(4 t)\right)$ is the modulation operator defined by the multiplication by $\exp \left(i|x|^{2} /(4 t)\right), D(t)$ is the dilation operator defined by $(D(t) \psi)(x)=(2 i t)^{-N / 2} \psi(x /(2 t))$, and $\mathcal{F}$ is the Fourier transform defined by $(\mathcal{F} \psi)(\xi)=(2 \pi)^{-N / 2} \int_{\mathbb{R}^{N}} e^{-i x \cdot \xi} \psi(x) d x$. For any $\rho, \rho^{\prime} \in \mathbb{R}, X_{\rho, \rho^{\prime}}=H^{\rho^{\prime}} \cap \mathcal{F}\left(H^{\rho}\right)$ denotes the weighted Sobolev space and $\mathcal{X}_{\rho, \rho^{\prime}}$ denotes the associated function space for solution to (1.1) defined by

$$
\begin{gathered}
\mathcal{X}_{\rho, \rho^{\prime}}=\left\{u \in C\left(\mathbb{R} ; X_{\rho, \rho^{\prime}}\right): u \in L_{\mathrm{loc}}^{q}\left(\mathbb{R} ; L^{r} \cap \dot{B}_{r}^{\rho^{\prime}}\right), M^{-1} u \in L_{\mathrm{loc}}^{q}\left(\mathbb{R} ; \dot{B}_{r}^{\rho^{\prime}}\right)\right. \\
\text { for any } q, r \text { with } 0 \leq 2 / q=\delta(r)<1\},
\end{gathered}
$$

where $\delta(r):=N(1 / 2-1 / r)$. A pair of numbers $(q, r)$ is called admissible if $2 \leq r \leq$ $2 N /(N-2)(2 \leq r \leq \infty$ if $N=1$ and $2 \leq r<\infty$ if $N=2)$, and $2 / q=\delta(r)$. The pair $(\infty, 2)$ is always admissible. For any $r$ with $2 \leq r \leq \infty$ and $T>0, Y^{r}(T)$ denotes the function space defined by

$$
Y^{r}(T):=\left\{u \in \S^{\prime}:\|u\|_{Y^{r}(T)}=\left\|D^{-1} M^{-1} u\right\|_{L^{\infty}\left([T, \infty) ; L^{r}\left(\mathbb{R}^{N}\right)\right)}<\infty\right\} .
$$

For any $\rho \in \mathbb{R}$ and any $T>0, \Sigma^{\rho}(T)$ denotes the function space defined as follows:

$$
\Sigma^{\rho}(T):=\left\{u \in \S^{\prime}:\|u\|_{\Sigma^{\rho}(T)}=\left\|D^{-1} M^{-1} u\right\|_{L^{\infty}\left([T, \infty) ; H^{\rho} r\left(\mathbb{R}^{N}\right)\right)}<\infty\right\} .
$$

The embedding $\Sigma^{\rho}(T) \hookrightarrow Y^{r}(T)$ holds for all $r \in[2, \infty]$ if $\rho>N / 2$, by the Sobolev embedding. The transform $v(t):=D^{-1}(t) M^{-1}(t) u(t)$ is equivalent to the pseudoconformal transform except for the inversion of time variable and was introduced in [8]. Moreover, the norm $\|u\|_{Y^{r}(T)}$ is equivalent to $\sup _{t \geq T}\left(t^{\delta(r)}\|u(t)\|_{L^{r}\left(\mathbb{R}^{N}\right)}\right)$, and the norm $\|u\|_{\Sigma^{\rho}(T)}$ is equivalent to the norm $\|u\|_{L^{\infty}\left([T, \infty) ; L^{2}\right)}+\left\||2 t|^{\rho} M(-t) u\right\|_{L^{\infty}\left([T, \infty) ; \dot{H}^{\rho}\right)}$, which is used in [4]. The $\|\cdot\|_{\Sigma^{\rho}(T)}$ norm is almost the same as the $\|\cdot\|_{\mathcal{X}_{\rho, \rho^{\prime}}}$ norm corresponding to the case $\rho^{\prime}=0$, $q=\infty$, and $r=2$.

Our results rely on the following existence result from [12] (see also [4]).

Proposition 1.1. (Proposition 1.5 in [12]) Let $\alpha$ and $N$ satisfy $\gamma(N)<\alpha<\alpha_{0}(N)$ and $N \geq 1$. Let $\phi \in X_{1,1}$ and let $u \in \mathcal{X}_{1,1}$ be the unique solution of (1.1) and (1.2) with $u(0)=$ $\phi$. Let $\phi_{ \pm} \in X_{1,1}$ be the corresponding asymptotic states at $t= \pm \infty$, respectively. Let $\rho$ satisfy $1 \leq \rho<(\alpha+1) \wedge 2$. Assume further that $\phi \in X_{\rho, 1}$. Then, $u \in \mathcal{X}_{\rho, 1}, \phi_{ \pm} \in X_{\rho, 1}$, and $U(-t) u(t) \rightarrow \phi_{ \pm}$in $X_{\rho, 1}$ as $t \rightarrow \pm \infty$. In particular, $u \in \Sigma^{\rho}(T)$ for $T>0$.

\subsection{Main result 1: the general case}

1.1.1. Asymptotics with $w_{n}$ for $X_{1,1}$, data. We begin with the construction of functions $w_{n}$ whose sums yield the asymptotics of the solution to (1.1) with (1.2). For an initial datum $\phi$ belonging to $X_{1,1}$, global existence, existence of the asymptotic states $\phi_{ \pm}$, and the asymptotic 
completeness in $X_{1,1}$ are known. For the construction of $w_{n}$, we first define the $(\lceil\alpha\rceil+1)$ linear operator $G$ by

$$
G\left(u_{1}, \ldots, u_{\lceil\alpha\rceil+1}\right)(t):=i \int_{t}^{\infty} U(t-s) \prod_{k=1}^{\lceil\alpha\rceil+1} u_{k}(s) d s,
$$

where $\lceil\alpha\rceil$ denotes the minimum integer larger than or equal to $\alpha$, that is,

$$
\lceil\alpha\rceil:=\min \{m \in \mathbb{Z}: m \geq \alpha\} .
$$

This is not the usual Gauss symbol $[\alpha]$ which denotes the maximum number smaller than or equal to $\alpha$. We denote $\alpha /\lceil\alpha\rceil$ by $\beta$. By the definition of $\lceil\alpha\rceil$, it always holds that $(\lceil\alpha\rceil-1) /\lceil\alpha\rceil<\beta \leq 1$. We note that (1.1) with (1.2) can be written as

$$
u=U(t) \phi_{+}+G\left(|u|^{\beta}, \ldots,|u|^{\beta}, u\right) .
$$

Now, the sequence of functions $\left\{w_{n}(t)\right\}_{n=0}$ is given in terms of $\phi_{+}$as follows:

$$
w_{0}=U(t) \phi_{+}, \quad w_{n}=\sum_{i_{1}+\cdots+i_{\lceil\alpha\rceil+1}=n-1} G\left(\widetilde{w}_{i_{1}}, \ldots, \widetilde{w}_{i_{\lceil\alpha\rceil}}, w_{i_{\lceil\alpha\rceil+1}}\right),
$$

where $\widetilde{w}_{0}=\left|w_{0}\right|^{\beta}$ and $\widetilde{w}_{n}=\left|\sum_{k=0}^{n} w_{k}\right|^{\beta}-\left|\sum_{k=0}^{n-1} w_{k}\right|^{\beta}$ for $n \geq 1$. Since all the indices in the definition of $w_{n}$ are smaller than $n, w_{n}$ is well defined. Each function $w_{n}$ takes a complicated abstract form. For example, $w_{1}(t)$ is equal to

$$
G\left(\widetilde{w}_{0}, \ldots, \widetilde{w}_{0}, w_{0}\right)=i \int_{t}^{\infty} U(t-s)\left|U(s) \phi_{+}\right|^{\alpha} U(s) \phi_{+} d s
$$

and $w_{2}(t)$ is equal to

$$
\begin{aligned}
& \sum_{i_{1}+\cdots+i_{\lceil\alpha\rceil+1}=1} G\left(\widetilde{w}_{i_{1}}, \ldots, \widetilde{w}_{i_{\lceil\alpha\rceil}}, w_{i_{\lceil\alpha\rceil+1}}\right) \\
= & i\lceil\alpha\rceil \int_{t}^{\infty} U\left(t-s_{1}\right)\left[\left|U\left(s_{1}\right) \phi_{+}\right|^{\alpha-\beta} U\left(s_{1}\right) \phi_{+}\right. \\
& \left.\times\left(\left.\left|\int_{s_{1}}^{\infty} U\left(s_{1}-s_{2}\right)\right| U\left(s_{2}\right) \phi_{+}\right|^{\alpha} U\left(s_{2}\right) \phi_{+} d s_{2}+\left.U\left(s_{1}\right) \phi_{+}\right|^{\beta}-\left|U\left(s_{1}\right) \phi_{+}\right|^{\beta}\right)\right] d s_{1} \\
& -\int_{t}^{\infty} U\left(t-s_{1}\right)\left(\int_{s_{1}}^{\infty} U\left(s_{1}-s_{2}\right)\left|U\left(s_{2}\right) \phi_{+}\right|^{\alpha} U\left(s_{2}\right) \phi_{+} d s_{2}\right)\left|U\left(s_{1}\right) \phi_{+}\right|^{\alpha} d s_{1} .
\end{aligned}
$$

Now, let us state our first main result. We use the notation $e(n):=\sum_{k=1}^{n} \beta^{k-1}$.

Theorem 1. Let $N \geq 1$. Let $\gamma(N)<\alpha<\alpha_{0}(N)$. Put $\theta=N \alpha / 2$. Let $\phi \in X_{1,1}$ and let $u \in \mathcal{X}_{1,1}$ be the unique solution to (1.1) and (1.2) with $u(0)=\phi$. Let $\phi_{+} \in X_{1,1}$ be the corresponding asymptotic states at $t=\infty$ and define $w_{n}$ as $(1.8)$. Let $I=[T, \infty)$ with $T \gg 1$. Then, it holds for all $r$ with $\delta(r) \in[0,(\theta-1) \wedge 1)$, admissible pairs $\left(q_{0}, r_{0}\right)$, and $n \geq 0$ that

$$
\begin{aligned}
&\left\|w_{n}\right\|_{Y^{r}(T)} \leq C T^{(1-\theta+(\delta(\alpha+2) \vee \delta(r))) e(n)}, \\
&\left\|w_{n}\right\|_{L^{q_{0}\left(I ; L^{r_{0}}\right)}} \leq C T^{(1-\theta+\delta(\alpha+2)) e(n)-\delta(\alpha+2) / 2},
\end{aligned}
$$


and

$$
\begin{aligned}
&\left\|u-\sum_{k=0}^{n} w_{k}\right\|_{Y^{r}(T)} \leq C T^{(1-\theta+(\delta(\alpha+2) \vee \delta(r))) e(n+1)}, \\
&\left\|u-\sum_{k=0}^{n} w_{k}\right\|_{L^{q_{0}\left(I ; L^{\left.r_{0}\right)}\right.}} \leq C T^{(1-\theta+\delta(\alpha+2)) e(n+1)-\delta(\alpha+2) / 2} .
\end{aligned}
$$

Moreover, if $\alpha$ is an integer then

$$
u=\sum_{n=0}^{\infty} w_{n} \quad \text { in } Y^{r}(T) \cap L^{q_{0}}\left(I ; L^{r_{0}}\right) .
$$

Remark 1.1. We have $\theta>1$ if and only if $\alpha>2 / N$. We have $\theta>1+\delta(\alpha+2)$ if and only if $\theta>\gamma(N)$. Note that $2 / N<\gamma(N)<4 / N<\alpha_{0}(N)$.

Remark 1.2. The powers of $T$ appearing in (1.9)-(1.12) depend on $e(n)=\sum_{k=1}^{n} \beta^{k-1}$ which satisfies $\lim _{n \rightarrow \infty} e(n)=(1-\beta)^{-1}$ if $\beta<1$ and $\lim _{n \rightarrow \infty} e(n)=\infty$ if $\beta=1$. Since $\beta=\alpha /\lceil\alpha\rceil$, it means that the sharpness of approximation strongly depends on the fractional part of the power $\alpha$. The upper bound of the accuracy goes to infinity as $\alpha$ tends to an integer from below. In particular, if the power is an integer then the approximation can be made accurate arbitrarily.

Remark 1.3. In [12], they construct a second approximate function $V_{+}$such that $\| u-$

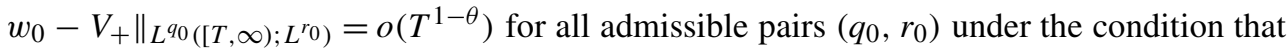
$\phi \in X_{\rho, 1}$ with $\rho=1$ in the case $N=1$ or the case where $N=2$ and $\alpha<2$, and with $\rho$ satisfying $\delta(2(\alpha+1)) \vee \delta((\alpha+1)(\alpha+2)) \vee(2 \delta(\alpha+2)) \vee 1<\rho<(\alpha+1) \wedge 2$ in the case $N=2$ and $\alpha \geq 2$ or the case $3 \leq N \leq 5$. Theorem 1 is better than this result in the following respects.

(1) There is no restriction on the space dimensions $N$, and we only need $\phi \in X_{1,1}$ for all $\alpha \in\left(\gamma(N), \alpha_{0}(N)\right)$.

(2) The best decay rate given by (1.12) is $(1-\theta+\delta(\alpha+2)) /(1-\beta)-\delta(\alpha+2) / 2$ if $\beta<1$, and it is $-\infty$ if $\beta=1$. This power becomes smaller than $(1-\theta)$ in the following cases: $\alpha>(1+\sqrt{89}) / 4 \in(\gamma(1), 3)$ if $N=1, \alpha>(-1+\sqrt{69}) / 4 \in(\gamma(2), 2)$ if $N=$ $2, \alpha>7 / 6 \in(\gamma(3), 2)$ if $N=3$, and $\alpha>\left(3-4 N+\sqrt{17 N^{2}+40 N+16}\right) / 4 N \in$ $(\gamma(N), 1)$ if $4 \leq N \leq 9$.

1.1.2. Sharp asymptotics for $N \leq 5$. The estimates in Theorem 1 are not sharp, while they hold for large $n$. Next we state more accurate estimates for $n=0,1,2$ under a suitable regularity condition on $\phi$.

Theorem 2. Let $1 \leq N \leq 5$ and $\alpha \in\left(\gamma(N), \alpha_{0}(N)\right)$. Put $\theta=\alpha N / 2$. Let $\rho$ satisfy $1 \leq \rho<$ $(\alpha+1) \wedge 2$ and

$$
\frac{N \alpha}{2(\alpha-\beta+1)} \vee\left(\frac{N}{2}-\frac{2(1-\beta)(\theta-1)}{\alpha}\right) \leq \rho .
$$

Let $\phi \in X_{\rho, 1}$ and let $u \in \mathcal{X}_{\rho, 1}$ be the unique solution to (1.1) and (1.2) with $u(0)=\phi$. Let $\phi_{+} \in X_{\rho, 1}$ be the corresponding asymptotic states at $t=\infty$ and define $w_{n}$ as (1.8). Let $r$ satisfy $0 \leq \delta(r)<(\theta-1) \wedge 1$ and $\left(q_{0}, r_{0}\right)$ be an admissible pair. Put $T \gg 1$ and 
$I=[T, \infty)$. Then, it holds that

$$
\left\|w_{n}\right\|_{Y^{r}(T)} \leq C T^{p+\delta(r)}, \quad\left\|w_{n}\right\|_{L^{q_{0}\left(I ; L^{\left.r_{0}\right)}\right.} \leq C T^{p}}
$$

for $n=0,1,2$, where $p=(1-\theta) e(n)$ and

$$
\left\|u-\sum_{k=0}^{n} w_{k}\right\|_{Y^{r}(T)} \leq C T^{q+\delta(r)}, \quad\left\|u-\sum_{k=0}^{n} w_{k}\right\|_{L^{q_{0}\left(I ; L^{\left.r_{0}\right)}\right.}} \leq C T^{q}
$$

for $n=0,1$, where $q=(1-\theta) e(n+1)$. Moreover, if $\alpha>1$ then

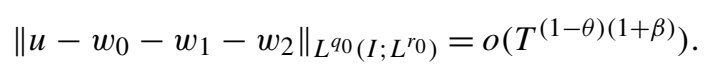

Remark 1.4. There exists $\rho \in[1,(\alpha+1) \wedge 2)$ so that (1.13) is satisfied in the following cases: $\alpha \in\left(\gamma(N), \alpha_{0}(N)\right)$ if $N \leq 3 ; \alpha \in\left(\gamma(4), \alpha_{0}(4)\right)$ and $\alpha \neq 1$ if $N=4$; and $\alpha \in$ $\left(1, \alpha_{0}(5)\right)$ if $N=5$.

Remark 1.5. Some estimates in Theorem 2 are sharp in such a sense that we have lower $L^{2}$ estimates. Indeed, Theorem 2 and Theorem 3 given below imply

$$
\begin{gathered}
\left\|w_{1}\right\|_{Y^{2}(T)} \geq\left\|V_{1}\right\|_{Y^{2}(T)}-\left\|w_{1}-V_{1}\right\|_{Y^{2}(T)}=c T^{1-\theta}, \\
\left\|u-w_{0}\right\|_{Y^{2}(T)} \geq\left\|w_{1}\right\|_{Y^{2}(T)}-\left\|u-w_{0}-w_{1}\right\|_{Y^{2}(T)}=c T^{1-\theta} .
\end{gathered}
$$

A similar argument also gives a lower $L^{2}$ bound for $w_{0}$. If $\alpha$ is even number then we have similar lower estimates on $w_{2}$ and $u-w_{0}-w_{1}$ (see Proposition 6.2).

1.1.3. The approximation of $w_{1}$. Next we consider the approximation of $w_{1}$ by $V_{1}$.

Theorem 3. Let $N \leq 8$ and $\alpha \in\left(\gamma(N), \alpha_{0}(N)\right)$. Put $\theta=\alpha N / 2$. Let $\rho$ satisfy $1 \leq \rho<$ $(\alpha+1) \wedge 2$ and

$$
\frac{\theta(1-\beta)+\beta}{\alpha-\beta+1} \vee \frac{\theta-\beta}{\alpha-\beta+1}<\rho .
$$

Let $\phi \in X_{\rho, 1}$ and let $u \in \mathcal{X}_{\rho, 1}$ be the unique solution to (1.1) and (1.2) with $u(0)=\phi$. Let $\phi_{+} \in X_{\rho, 1}$ be the corresponding asymptotic states at $t=\infty$ and define $w_{n}$ as (1.8). Let $r \geq 2$ with $\delta(r)<(\theta-1) \wedge 1$ and $\left(q_{0}, r_{0}\right)$ be an admissible pair. For $r$ and $r_{0}$ satisfying $\delta((\alpha+1) r) \leq \rho$ and $\delta\left((\alpha+1) r_{0}\right) \leq \rho$, it holds that

$$
\begin{gathered}
\left\|w_{1}-V_{1}\right\|_{Y^{r}(T)}=o\left(T^{1-\theta+\delta(r)}\right), \\
\left\|u-w_{0}-V_{1}\right\|_{Y^{r}(T)}=o\left(T^{1-\theta+\delta(r)}\right), \\
\left\|w_{1}-V_{1}\right\|_{L^{q_{0}\left(I ; L^{r_{0}}\right)}=o\left(T^{1-\theta}\right),}\left\|u-w_{0}-V_{1}\right\|_{L^{q_{0}\left(I ; L^{r_{0}}\right)}}=o\left(T^{1-\theta}\right),
\end{gathered}
$$

where

$$
V_{1}(t)=\frac{i t^{1-\theta}}{2^{\theta}(\theta-1)} M(t) D(t)\left|\mathcal{F} \phi_{+}\right|^{\alpha} \mathcal{F} \phi_{+} .
$$

Remark 1.6. There exists $\rho \in[1,(\alpha+1) \wedge 2)$ so that (1.14) is satisfied in the following cases: $\alpha \in\left(\gamma(N), \alpha_{0}(N)\right)$ if $N \leq 6$ and $\alpha \in(\gamma(N), 2 /(N-4))$ if $N=7,8$. We also note that (1.15)-(1.18) hold at least for $r=r_{0}=2$ since the condition $\delta((\alpha+1) 2)=$ $N \alpha / 2(\alpha+1)<\rho$ is weaker than (1.14). 


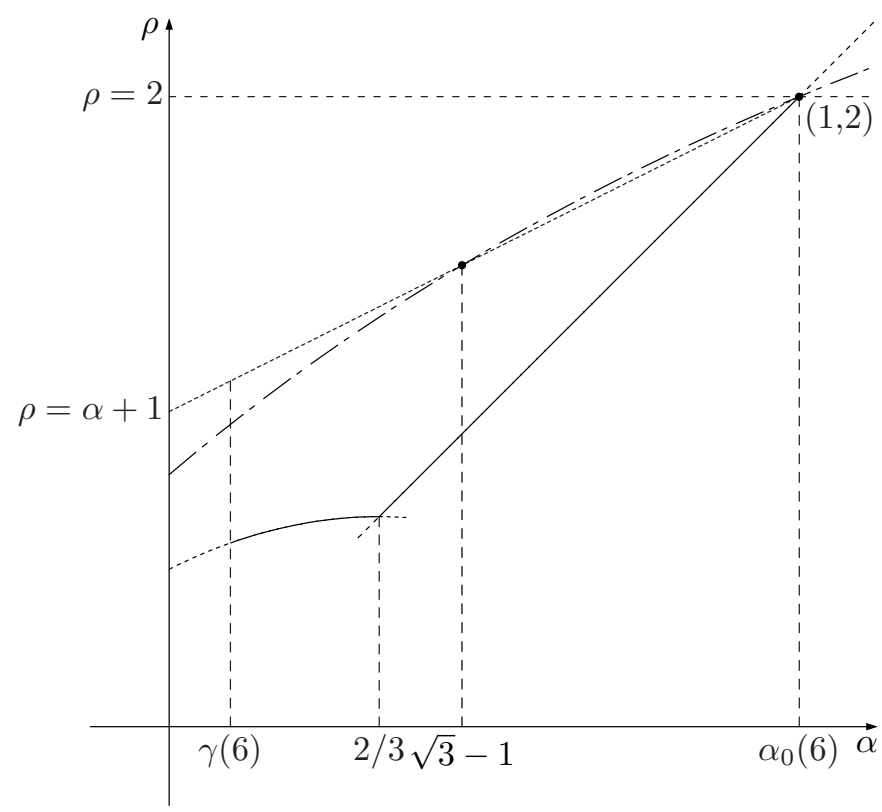

FIgURE 1. Admissible value of $\rho$ for Theorem 3 in the case $N=6$.

Remark 1.7. The condition (1.14) is needed for the estimate of $u-w_{0}-w_{1}$. If (1.14) is satisfied then the $Y^{r}(T)$ norm and $L^{q_{0}}\left(I ; L^{r_{0}}\right)$ norm of $u-w_{0}-w_{1}$ become the same order as (1.16) and (1.18), respectively (see the second part of Proposition 4.1). On the other hand, we need another condition when we estimate $w_{1}-V_{1}$. It is $\delta((\alpha+1) r) \leq$ $\rho$. In [12], they require $f\left(\mathcal{F} \phi_{+}\right) \in L^{r} \cap \dot{H}_{r^{\prime}}^{2 \delta(r)+\varepsilon}$ for the same estimate, while here we require only $f\left(\mathcal{F} \phi_{+}\right) \in \dot{H}^{\delta(r)}$. This is an improvement. It is due to Proposition 2.5 (see Remark 2.1). Figure 1 shows the conditions of Theorem 3 in the case $N=6$. Note that $\gamma(6)=(\sqrt{7}-1) / 3=0.548 \ldots$ and $\alpha_{0}(6)=1$. The solid curve shows the left-hand side of (1.14). Theorem 3 holds for $\alpha$ between this line and $\rho=\alpha+1$ (dotted curve). What is proved in [12] is the asymptotics (1.18) with $\left(q_{0}, r_{0}\right)=(\infty, 2),(2 / \delta(\alpha+2), \alpha+2)$ (and therefore with any admissible pair satisfying $\left.2 \leq r_{0} \leq \alpha+2\right)$. The dash-dotted curve is the graph of $\rho=\delta((\alpha+1)(\alpha+2))$. We see that we can choose $\rho \geq \delta((\alpha+1)(\alpha+2))$ only in the case $\alpha<\sqrt{3}-1$. Namely, (1.18) with $\left(q_{0}, r_{0}\right)=(2 / \delta(\alpha+2), \alpha+2)$ does not hold if $\alpha \geq \sqrt{3}-1$. Our Theorem 3 relaxes the regularity condition on data so that the asymptotics (1.18) holds for some $r_{0} \geq 2$ beyond $\alpha=\sqrt{3}-1$.

\subsection{Main result 2: the case where $\alpha$ is an even number}

By the fact that $|u|^{\alpha} u=u^{a} \bar{u}^{b}$ with $a, b \in \mathbb{N}$, we modify the definition of the $(\alpha+1)$-linear operator $G$ as follows:

$$
H\left(u_{1}, \ldots, u_{\alpha+1}\right)(t)=i \int_{t}^{\infty} U(t-s) u_{1} \cdots u_{\alpha / 2+1} \overline{u_{\alpha / 2+2}} \cdots \overline{u_{\alpha+1}}(s) d s .
$$


With this modified $\mathbb{R}$-multilinear operator $H,(1.1)$ with (1.2) can be written as

$$
u(t)=U(t) \phi_{+}+H(u, \ldots, u) .
$$

We define the function $w_{n}(t)$ as follows:

$$
w_{0}(t)=U(t) \phi_{+}, \quad w_{n}(t)=\sum_{i_{1}+\cdots+i_{\alpha+1}=n-1} H\left(w_{i_{1}}, \ldots, w_{i_{\alpha+1}}\right),
$$

and also define the function $V_{n}(t)$ as follows:

$$
V_{n}(t)=\frac{(i)^{n}}{n !} \cdot \frac{t^{n(1-\theta)}}{2^{n \theta}(\theta-1)^{n}} M(t) D(t)\left|\mathcal{F} \phi_{+}\right|^{\alpha n} \mathcal{F} \phi_{+} .
$$

We use the following notation: $a \asymp b$ holds if there exist positive constants $c_{1}$ and $c_{2}$ such that $c_{1} b \leq a \leq c_{2} b$.

THEOREM 4. Let $N=1,2,3$. Let $\alpha$ be a positive even number satisfying $\gamma(N)<\alpha<$ $\alpha_{0}(N)$. Put $\theta=N \alpha / 2$. Let $\rho$ satisfy $\rho=1$ if $N=1$ and $N / 2<\rho<2$ if $N=2$, 3. Let $\phi \in X_{\rho, 1}$ and let $u \in \mathcal{X}_{\rho, 1}$ be the unique solution to (1.1) and (1.2) with $u(0)=\phi$. Let $\phi_{+} \in X_{\rho, 1}$ be the corresponding scattered state at $t=\infty$ and define $w_{n}$ and $V_{n}$ as (1.21) and (1.22), respectively. Then,

$$
\begin{gathered}
\left\|w_{n}\right\|_{\Sigma^{\rho}(T)} \asymp T^{n(1-\theta)}, \\
\left\|u-\sum_{k=0}^{n} w_{k}\right\|_{\Sigma^{\rho}(T)} \asymp T^{(n+1)(1-\theta)},
\end{gathered}
$$

and

$$
\begin{gathered}
\left\|V_{n}\right\|_{\Sigma^{\rho}(T)}=C T^{n(1-\theta)}, \\
\left\|w_{n}-V_{n}\right\|_{\Sigma^{\rho}(T)}=o\left(T^{n(1-\theta)}\right)
\end{gathered}
$$

as $T \rightarrow \infty$. Moreover, if $T$ is sufficiently large then

$$
u=\sum_{k=0}^{\infty} w_{k} \quad \text { in } \Sigma^{\rho}(T) .
$$

Furthermore, the above asymptotics hold with $\|\cdot\|_{Y^{r}(T)}$ for any $r \in[2, \infty]$.

Remark 1.8. In Theorem 4, the admissible value of $\alpha$ is $\alpha=4,6,8, \ldots$ if $N=1, \alpha=$ $2,4,6, \ldots$ if $N=2$, and $\alpha=2$ if $N=3$. This is because $\gamma(1)=(1+\sqrt{17}) / 2 \in(2,3)$, $\gamma(2)=\sqrt{2}, \gamma(3)=1, \alpha_{0}(1)=\alpha_{0}(2)=\infty$, and $\alpha_{0}(3)=4$. We also note that $\alpha_{0}(N) \leq 2$ for $N \geq 4$. Therefore, there is no even number in $\left(\gamma(N), \alpha_{0}(N)\right)$ if $N \geq 4$.

Remark 1.9. The asymptotics in Theorem 4 can be summarized as follows:

$$
\begin{aligned}
& u(t)=w_{0}(t)+w_{1}(t)+\cdots+w_{n}(t)+\cdots \\
& V_{0}(t) \quad V_{1}(t) \quad V_{n}(t)
\end{aligned}
$$

The true solution $u$ is expanded as the infinite sum of $w_{n}$, and each $w_{n}$ is approximated by $V_{n}$. We also note that the sums of $V_{n}$ do not necessarily yield the approximate solution. 
Remark 1.10. Our regularity condition is required for the control of the $L^{\infty}$ norm of $\mathcal{F} \phi_{+}$with the Sobolev embedding. It seems to be natural for higher-order approximations. For example, to obtain the upper bound of $\left\|V_{n} ; L^{r}\right\|$, we need the boundedness of $\left\|\mathcal{F} \phi_{+} ; L^{r(\alpha n+1)}\right\|$. Hence, it is almost inevitable that $\mathcal{F} \phi_{+}$belongs to $L^{\infty}$ if we are concerned with large $n$. Then, we assume $\phi_{+} \in X_{\rho, 1}$ with $\rho>N / 2$ which ensures the desired $L^{\infty}$ boundedness of $\mathcal{F} \phi_{+}$and, thanks to this assumption, we can estimate the $L^{r}$ norm of the solution $u$, approximate solutions $w_{n}$ and $V_{n}$, and their distance for all $2 \leq r \leq \infty$.

Remark 1.11. The construction of approximate solution $w_{n}$ is based on the multilinearity of the nonlinearity. It is applicable to the equation of the form $u=w_{0}+F(u)$, where $F$ is a multilinear function (see Theorem 3.2). A typical example of the equation of this form is a Cauchy problem for a nonlinear partial differential equation $\partial_{t} u+L u+f(u)=0$ with $u(0, x)=u_{0}(x)$, where $(t, x) \in \mathbb{R}^{1+N}, L$ is a linear (differential) operator, and $f$ is a nonlinear function. With the virtue of the Duhamel principle, this equation is, at least formally, written as

$$
u(t)=e^{-t L} u_{0}-\int_{0}^{t} e^{-(t-s) L} f(u(s)) d s .
$$

Examples of multilinear nonlinearity other than $|u|^{\alpha} u$ with positive even $\alpha$ are the Hartree type nonlinearity $\left(|x|^{-\gamma} *|u|^{2}\right) u$ and $\partial_{x}\left(u^{2}\right)$ which appears in Korteweg-de Vries (KdV) equations. In [14], the author applies this construction to the semi-classical nonlinear Schrödinger equation with Hartree-type nonlinearity.

The rest of the paper is constructed as follows. In Section 2, we first collect preliminary estimates. Sections 3 and 4 are devoted to the study of approximation by $w_{n}$. We first state abstract results of asymptotic expansion in Section 3, and then, applying them, we prove Theorem 1 and some results in Theorem 4. We also prove Theorem 2 in Section 4 without the abstract results. We study the asymptotic behavior of $w_{n}$ in Sections 5 and 6. In Section 5, we see the derivation of the function $V_{n}$ by a formal observation, and we finally prove Theorems 3 and the rest of the results in Theorem 4.

\section{Preliminary estimates}

\subsection{Estimates for the proof of Theorems 1 and 2}

LemMA 2.1. Let $N \geq 1$ and $\alpha>2 / N$. Let $G=G\left(u_{1}, \ldots, u_{\lceil\alpha\rceil+1}\right)$ be the $(\lceil\alpha\rceil+1)$-linear operator defined by (1.6). Put $\theta=N \alpha / 2$ and let $r \in[2, \infty]$ satisfy $0 \leq \delta(r)<1$ and $\delta(r)<$ $\theta-1$. It holds that

$$
\|G\|_{Y^{r}(T)} \leq C T^{1-\theta+\delta(r)}\left(\prod_{k=1}^{\lceil\alpha\rceil}\left\|\left|u_{k}\right|^{1 / \beta}\right\|_{Y^{s_{k}(T)}}^{\beta}\right)\left\|u_{\lceil\alpha\rceil+1}\right\|_{Y^{s\lceil\alpha\rceil+1}(T)},
$$

where $s_{k} \in[2, \infty](k=1, \ldots,\lceil\alpha\rceil+1)$ satisfy

$$
\beta \sum_{k=1}^{\lceil\alpha\rceil} \frac{1}{s_{k}}+\frac{1}{s_{\lceil\alpha\rceil+1}}+\frac{1}{r}=1 .
$$


Proof. From the $L^{p}-L^{q}$ estimate and the Hölder inequality, we obtain

$$
\begin{aligned}
\|G\|_{L^{r}} & \leq C \int_{t}^{\infty}|t-s|^{-\delta(r)}\left\|\prod_{k=1}^{\lceil\alpha\rceil+1} u_{k}(s)\right\|_{L^{r^{\prime}}} d s \\
& \leq C \int_{t}^{\infty}|t-s|^{-\delta(r)}\left(\prod_{k=1}^{\lceil\alpha\rceil}\left\|\left|u_{k}(s)\right|^{1 / \beta}\right\|_{L^{s k}}^{\beta}\right)\left\|u_{\lceil\alpha\rceil+1}(s)\right\|_{L^{s\lceil\alpha\rceil+1}} d s,
\end{aligned}
$$

where $r \in[2, \infty]$ and $s_{k}$ satisfy (2.2). By the definition of $Y^{r}(T)$, the right-hand side is bounded by

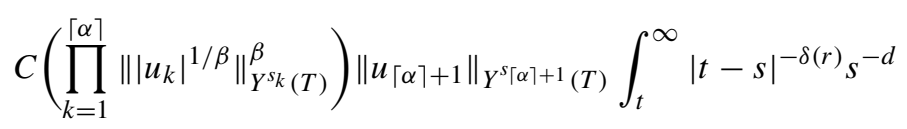

for any $t \geq T$, where

$$
\begin{aligned}
d=\beta \sum_{k=1}^{\lceil\alpha\rceil} \delta\left(s_{k}\right)+\delta\left(s_{\lceil\alpha\rceil+1}\right) & =\frac{N(\alpha+2)}{2}-N\left(\beta \sum_{k=1}^{\lceil\alpha\rceil} \frac{1}{s_{k}}-\frac{1}{s_{\lceil\alpha\rceil+1}}+\frac{1}{r}\right)-\delta(r) \\
& =\theta-\delta(r)
\end{aligned}
$$

by the definition of $\delta\left(s_{k}\right)$ and $\delta(r)$. The time integral is equal to $C t^{1-\theta}$ with some positive constant $C$ if $\delta(r) \in[0,1)$ and $\delta(r)<\theta-1$. Again by the definition of the space $Y^{r}(T)$ we obtain the result.

LeMmA 2.2. Let $N \geq 1$ and $\alpha>2 / N$. Let $G=G\left(u_{1}, \ldots, u_{\lceil\alpha\rceil+1}\right)$ be the $(\lceil\alpha\rceil+1)$-linear operator defined by (1.6). Put $\theta=N \alpha / 2$. Let $\left(q_{0}, r_{0}\right)$ be an admissible pair. Put $\theta=N \alpha / 2$ and $I=[T, \infty)$. Suppose that $r \in[2,2 N /(N-2)](r \in[2, \infty]$ if $N=1, r \in[2, \infty)$ if $N=2)$ and $s_{k} \in[2, \infty]$ satisfy (2.2). Then, it holds that

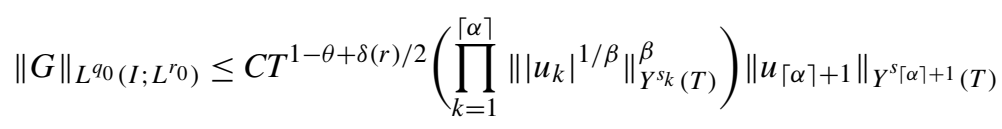

if $\delta(r)<2(\theta-1)$. Moreover, for a subset $K \subset\{1,2, \ldots,\lceil\alpha\rceil\}$ and $d_{K}=\beta \sum_{k \in K} \delta\left(s_{k}\right) / 2$, it holds that

$$
\begin{aligned}
\|G\|_{L^{q_{0}\left(I ; L^{\left.r_{0}\right)} \leq\right.}} & C T^{1-\theta+\delta(r) / 2+d_{K}}\left(\prod_{k \in K}\left\|\left|u_{k}\right|^{1 / \beta}\right\|_{L^{2 / \delta\left(s_{k}\right)}\left(I ; L^{\left.s_{k}\right)}\right.}^{\beta}\right) \\
& \times\left(\prod_{k \notin K}\left\|\left|u_{k}\right|^{1 / \beta}\right\|_{Y^{s_{k}(T)}}^{\beta}\right)\left\|u_{\lceil\alpha\rceil+1}\right\|_{Y^{s\lceil\alpha\rceil+1}(T)}
\end{aligned}
$$

if $\delta(r) / 2+d_{K}<\theta-1$, and that

$$
\begin{aligned}
\|G\|_{L^{q_{0}\left(I ; L^{\left.r_{0}\right)} \leq\right.}} & C T^{1-\theta+\delta(r) / 2+d_{K}+\delta(\lceil\alpha\rceil+1) / 2}\left(\prod_{k \in K}\left\|\left|u_{k}\right|^{1 / \beta}\right\|_{L^{2 / \delta\left(s_{k}\right)}\left(I ; L^{s k}\right)}^{\beta}\right) \\
& \times\left(\prod_{k \notin K}\left\|\left|u_{k}\right|^{1 / \beta}\right\|_{Y^{s_{k}(T)}}^{\beta}\right)\left\|u_{\lceil\alpha\rceil+1}\right\|_{L^{2 / \delta\left(s_{\lceil\alpha\rceil+1}\right)}\left(I ; L^{s}[\alpha]+1\right)}
\end{aligned}
$$

if $\delta(r) / 2+d_{K}+\delta\left(s_{\lceil\alpha\rceil+1}\right) / 2<\theta-1$.

This lemma is shown in a similar way to the previous lemma. We use the Strichartz estimate instead of the $L^{p}-L^{q}$ estimate. 


\subsection{Estimates for the proof of Theorem 4}

In this section, we assume that $\alpha$ is an even integer.

LemmA 2.3. Let $N=1,2,3$. Let $\alpha \in\left(\gamma(N), \alpha_{0}(N)\right)$ be an even integer. Let $H=$ $H\left(u_{1}, \ldots, u_{\alpha+1}\right)$ be the $(\alpha+1)$-linear operator defined by (1.19). Let $\rho=1$ if $N=1$ and $\rho \in(N / 2,2)$ if $N=2,3$. Then, it holds for all $T>0$ that

$$
\|H\|_{\Sigma^{\rho}(T)} \leq C_{1} T^{1-\theta} \prod_{k=1}^{\alpha+1}\left\|u_{k}\right\|_{\Sigma^{\rho}(T)}
$$

where $\theta=N \alpha / 2$.

Proof. By the definition of $H$, we calculate

$$
\begin{aligned}
& D^{-1}(t) M^{-1}(t) H(t) \\
& \quad=i D^{-1}(t) M^{-1}(t) \int_{t}^{\infty} U(t-s) u_{1} \cdots u_{\alpha / 2+1} \overline{u_{\alpha / 2+2}} \cdots \overline{u_{\alpha+1}}(s) d s .
\end{aligned}
$$

Let us note that $D^{-1}(t) M^{-1}(t) U(t-s)=U(1 / 4 s-1 / 4 t) D^{-1}(s) M^{-1}(s)$. Then, it holds that

$$
\left\|D^{-1}(t) M^{-1}(t) H(t)\right\|_{H^{\rho}} \leq C \int_{t}^{\infty}|2 s|^{-N \alpha / 2} \prod_{k=1}^{\alpha+1}\left\|D^{-1}(s) M^{-1}(s) u_{k}\right\|_{H^{\rho}} d s,
$$

where we use the fact that the nonlinearity is Gauge-invariant and that the $H^{\rho}$ is an algebra for $\rho>N / 2$. Taking the $L^{\infty}$ norm in time, we obtain the result.

\subsection{Estimates for the approximation of $w_{n}$}

Next we show the error estimates which are used when we estimate $w_{n}-V_{n}$. We use these results for the proof of Theorems 3 and 4 .

LEMMA 2.4. Let $U(t)=e^{i t \Delta}$ and $r \in[2, \infty)$. There exists a real-valued continuous function $h$ with $h(0)=0$ such that

$$
\|(U(t)-1) \phi\|_{L^{r}} \leq C h(t)
$$

if $\phi \in \dot{H}^{\delta(r)}$. Moreover, it holds for any $\phi \in H^{s}$ with $s>N / 2$ that

$$
\|(U(t)-1) \phi\|_{L^{\infty}} \leq C h(t) .
$$

Proof. The first estimate with $r=2$ is clear because $\|(U(t)-1) f\|_{L^{2}}$ is bounded by $2\|f\|_{L^{2}}$ and tends to zero as $t \rightarrow 0$. Since $U(t)-1$ commutes with both $(-\Delta)^{s / 2}$ and $(1-\Delta)^{s / 2}$, the same argument shows, for any $s \in \mathbb{R}$, that the norms $\|(U(t)-1) \phi\|_{\dot{H}^{s}}$ and $\|(U(t)-1) \phi\|_{H^{s}}$ tend to zero if $\phi$ belongs to $\dot{H}^{s}$ and $H^{s}$, respectively. Then, the results follow from the embedding $\dot{H}^{\delta(r)} \hookrightarrow L^{r}(r \in[2, \infty))$ and $H^{s} \hookrightarrow L^{\infty}(s>N / 2)$.

Remark 2.1. In [12], they use the estimate

$$
\|(U(t)-1) \psi\|_{L^{r}} \leq \begin{cases}C t^{\delta(r)}\|\psi\|_{\dot{H}_{r^{\prime}}^{4 \delta(r)}}, & \text { if } N=1, \\ C t^{\varepsilon \delta(r) /(1-\varepsilon)}\|\psi\|_{\dot{H}_{r^{\prime}}^{2 \delta(r) /(1-\varepsilon)},}, & \text { if } N \geq 2,\end{cases}
$$


where $\varepsilon>0$ and $r$ satisfies $0 \leq \delta(r) \leq 1-\varepsilon$. The estimates in Lemma 2.4 are improvements of them and relax the regularity conditions on data.

For a real number $\sigma \geq 1$ and a spacetime function $\phi_{+}$, we define the error term $E_{\sigma}$ as

$$
E_{\sigma}(t):=M(t) D(t) i \int_{t}^{\infty}(U(1 / 4 s-1 / 4 t)-1) s^{\sigma(1-\theta)-1}\left|\mathcal{F} \phi_{+}\right|^{\alpha \sigma} \mathcal{F} \phi_{+} d s .
$$

Proposition 2.5. Let $N \geq 1, \alpha>2 / N$, and $\sigma \geq 1$. Let $E_{\sigma}$ be defined by (2.7). Let $\mathcal{F} \phi_{+} \in$ $H^{\rho}$ for some $\rho$ with $1 \leq \rho<(\alpha+1) \wedge 2$. Let $r \in[2, \infty]$. Suppose that $\rho$ satisfy

$$
\rho \geq \delta(r(\alpha \sigma+1)) \quad \text { if } r<\infty, \quad \rho>\frac{N}{2} \quad \text { if } r=\infty .
$$

Then, there exists a continuous function $h: \mathbb{R}_{\geq 0} \rightarrow \mathbb{R}_{\geq 0}$ with $h(0)=0$ such that

$$
\begin{aligned}
\left\|E_{\sigma}\right\|_{Y^{r}(T)} & \leq h(1 / T) T^{\sigma(1-\theta)}\left\|\mathcal{F} \phi_{+}\right\|_{H^{\rho}}^{1+\alpha \sigma}, \\
\left\|E_{\sigma}\right\|_{L^{q}\left(I ; L^{r}\right)} & \leq h(1 / T) T^{\sigma(1-\theta)-\delta(r) / 2}\left\|\mathcal{F} \phi_{+}\right\|_{H^{\rho}}^{1+\alpha \sigma},
\end{aligned}
$$

where $q=2 / \delta(r)$ and $I=[T, \infty)$.

Proof. By the definition of $E_{\sigma}$, we have

$$
\left\|E_{\sigma}(t)\right\|_{L^{r}} \leq C t^{-\delta(r)} \int_{t}^{\infty} s^{\sigma(1-\theta)-1}\left\|\mathcal{U}(t, s)\left|\mathcal{F} \phi_{+}\right|^{\alpha \sigma} \mathcal{F} \phi_{+}\right\|_{L^{r}} d s,
$$

where $\mathcal{U}(t, s):=U(1 / 4 s-1 / 4 t)-1$. If the $L^{r}$ norm in the right-hand side is bounded by $h(1 / s)\left\|\mathcal{F} \phi_{+}\right\|_{H^{\rho}}^{1+\alpha \sigma}$ for some continuous function $h$ with $h(0)=0$, we obtain the result. Hence, we prove this. Applying Lemma 2.4, we obtain

$$
\left\|\mathcal{U}(t, s)\left|\mathcal{F} \phi_{+}\right|^{\alpha \sigma} \mathcal{F} \phi_{+}\right\|_{L^{r}} \leq h\left((1 / 4 s-1 / 4 t)\left\|\left|\mathcal{F} \phi_{+}\right|^{\alpha \sigma} \mathcal{F} \phi_{+}\right\|_{\dot{H}^{\delta(r)}}\right.
$$

for $N \geq 1$ and $r \in[2, \infty)$. Moreover, we use the estimate

$$
\begin{aligned}
\left\|\left|\mathcal{F} \phi_{+}\right|^{\alpha \sigma} \mathcal{F} \phi_{+}\right\|_{\dot{H}^{\delta(r)}} & \leq C\left\|\left|\mathcal{F} \phi_{+}\right|^{\alpha \sigma} \mathcal{F} \phi_{+}\right\|_{B_{2}^{\delta(r)}} \leq C\left\|\mathcal{F} \phi_{+}\right\|_{L^{p}}^{\alpha \sigma}\left\|\mathcal{F} \phi_{+}\right\|_{B_{q}^{\delta(r)}} \\
& \leq C\left\|\mathcal{F} \phi_{+}\right\|_{\dot{H}^{\delta(p)}}^{\alpha \sigma}\left\|\mathcal{F} \phi_{+}\right\|_{H^{\delta(r)+\delta(q)}}
\end{aligned}
$$

for any $p, q \geq 2$ satisfying $\alpha \sigma / p+1 / q=1 / 2$, that is, $\alpha \sigma \delta(p)+\delta(q)=N \alpha \sigma / 2$, where we have used the usual embeddings between Besov and Triebel-Lizorkin spaces and nonlinear estimates in homogeneous Besov spaces [4]. We now choose $p$ so that $\delta(p)=\delta(r)+\delta(q)$. Then,

$$
\delta(p)=\frac{N \alpha \sigma}{2(\alpha \sigma+1)}+\frac{\delta(r)}{\alpha \sigma+1}=\delta(r(\alpha \sigma+1))
$$

since $\alpha \sigma \delta(p)+\delta(q)=N \alpha \sigma / 2$. Thus, if $\delta(p) \leq \rho$ then we conclude that

$$
\left\|\mathcal{F} \phi_{+}\right\|_{\dot{H}^{\delta(p)}}^{\alpha \sigma}\left\|\mathcal{F} \phi_{+}\right\|_{H^{\delta(r)+\delta(q)}} \leq\left\|\mathcal{F} \phi_{+}\right\|_{H^{\rho}}^{\alpha \sigma+1} .
$$

Similarly, if $\rho>N / 2$ then we have

$$
\begin{aligned}
\left\|\mathcal{U}(t, s)\left|\mathcal{F} \phi_{+}\right|^{\alpha \sigma} \mathcal{F} \phi_{+}\right\|_{L^{\infty}} & \leq C h(1 / 4 s-1 / 4 t)\left\|\left|\mathcal{F} \phi_{+}\right|^{\alpha \sigma} \mathcal{F} \phi_{+}\right\|_{H^{\rho}} \\
& \leq C h(1 / 4 s-1 / 4 t)\left\|\mathcal{F} \phi_{+}\right\|_{H^{\rho}}^{\alpha \sigma+1},
\end{aligned}
$$

which completes the proof. 
Proposition 2.6. Let $N=1,2,3, \alpha>2 / N$ be an even integer, and $n$ be a non-negative integer. Let $E_{n}$ be defined by (2.7) with $\sigma=n$. Let $\mathcal{F} \phi_{+} \in H^{\rho}$ for some $\rho$ with $N / 2<\rho<2$. Then, there exists a continuous function $h: \mathbb{R}_{\geq 0} \rightarrow \mathbb{R}_{\geq 0}$ with $h(0)=0$ such that

$$
\left\|E_{n}\right\|_{\Sigma^{\rho}(T)} \leq C h(1 / T) T^{n(1-\theta)}\left\|\mathcal{F} \phi_{+}\right\|_{H^{\rho}}^{\alpha n+1} .
$$

Proof. By the definition of $E_{n}$, we deduce that

$$
\begin{aligned}
\left\|D^{-1}(t) M(-t) E_{n}(t)\right\|_{H^{\rho}} & \leq \int_{t}^{\infty}\left\|\mathcal{U}(t, s) s^{n(1-\theta)-1}\left|\mathcal{F} \phi_{+}\right|^{\alpha n} \mathcal{F} \phi_{+}\right\|_{H^{\rho}} d s \\
& \leq C h(1 / 4 t) t^{n(1-\theta)}\left\|\left|\mathcal{F} \phi_{+}\right|^{\alpha n} \mathcal{F} \phi_{+}\right\|_{H^{\rho}} \\
& \leq C h(1 / 4 t) t^{n(1-\theta)}\left\|\mathcal{F} \phi_{+}\right\|_{H^{\rho}}^{\alpha n+1} .
\end{aligned}
$$

\section{Abstract results}

In this section, we consider an abstract semilinear equation with a $k$-linear nonlinearity for some $k \geq 2$. Specifically, we consider the abstract equation

$$
u=L(f)+N_{k}(u, \ldots, u),
$$

where the initial data $f$ take values in some data space $D$, the solution $u$ takes values in some solution space $S$, the linear operator $L: D \rightarrow S$ is densely defined, and the $k$-linear operator $N_{k}: S \times \cdots \times S \rightarrow S$ is also densely defined.

Assumption 3.1. Let $\left(D,\|\cdot\|_{D}\right)$ be a Banach space of initial data, and $\left(S,\|\cdot\|_{S}\right)$ be a Banach space of spacetime functions. The operators $L$ and $N$ satisfy

$$
\|L(f)\|_{S} \leq C_{0}\|f\|_{D}
$$

and

$$
\left\|N_{k}\left(u_{1}, \ldots, u_{k}\right)\right\|_{S} \leq \varepsilon \prod_{l=1}^{k}\left\|u_{l}\right\|_{S}
$$

with positive constants $C_{0}$ and $\varepsilon$.

This assumption is the same as the 'quantitative well-posedness' introduced by Bejenaru and Tao. For the well-posedness result of (3.1), see [1]. We adopt the well-posedness result into the approximation of the solution to (3.1).

THEOREM 3.2. Assume that (3.1) satisfies Assumption 3.1. Let $u$ be a solution of (3.1) with $\|u\|_{S} \leq C_{1}\|f\|_{D}$ with some constant $C_{1}$. If we define the approximate solution $w_{n}$ by the recursive formula

$$
w_{0}:=L(f), \quad w_{n}:=\sum_{\substack{i_{1}, \ldots, i_{k} \geq 0 \\ i_{1}+\cdots+i_{k}=n-1}} N_{k}\left(w_{i_{1}}, \ldots, w_{i_{k}}\right),
$$

then we have the following boundedness and asymptotics:

$$
\begin{gathered}
\left\|w_{n}\right\|_{S} \leq I(n, k) \varepsilon^{n}\left(C_{0}\|f\|_{D}\right)^{n(k-1)} \\
\left\|u-\sum_{l=0}^{n} w_{l}\right\|_{S} \leq I(n+1, k) \varepsilon^{n+1}\left(\left(C_{0} \vee C_{1}\right)\|f\|_{D}\right)^{(n+1)(k-1)},
\end{gathered}
$$


where $I(n, k)$ is the generalized Catalan number defined by

$$
I(n, k)=\frac{(k n) !}{(n(k-1)+1) ! n !} .
$$

Moreover, if $\varepsilon$ is sufficiently small then $w_{n}$ gives the absolutely convergent expansion

$$
u=\sum_{n=0}^{\infty} w_{n} \text { in } S .
$$

We illustrate the proof below, because we quote some part of it later.

Proof. Let $I(n, k)$ be a sequence which satisfies $I(0, k)=1$ and

$$
I(n, k)=\sum_{i_{1}+\cdots+i_{k}=n-1} \prod_{m=1}^{k} I\left(i_{m}, k\right) .
$$

We first prove the boundedness of $w_{n}$ by induction. If (3.2) holds for $n=0,1, \ldots, n_{0}-1$, then

$$
\begin{aligned}
\left\|w_{n_{0}}\right\|_{S} & =\left\|\sum_{i_{1}+\cdots+i_{k}=n_{0}-1} N_{k}\left(w_{i_{1}}, \ldots, w_{i_{k}}\right)\right\|_{S} \\
& \leq \varepsilon^{n_{0}}\left(C_{0}\|f\|_{D}\right)^{n(k-1)} \sum_{i_{1}+\cdots+i_{k}=n_{0}-1} \prod_{m=1}^{k} I\left(i_{m}, k\right) \\
& =I\left(n_{0}, k\right) \varepsilon^{n_{0}}\left(C_{0}\|f\|_{D}\right)^{n_{0}(k-1)} .
\end{aligned}
$$

Therefore, it holds for $n=n_{0}$, and for all $n \geq 0$. We next prove the asymptotics (3.3). An elementary computation shows that

$$
\begin{aligned}
u-\sum_{l=0}^{n} w_{l} & =N_{k}(u, \ldots, u)-\sum_{l=1}^{n} w_{l} \\
& =\sum_{m=1}^{k} \sum_{i_{m}+\cdots+i_{k}=n-1} N_{k}\left(u, \ldots, u, u-\sum_{l=0}^{i_{m}} w_{l}, w_{i_{m+1}}, \ldots, w_{i_{k}}\right) .
\end{aligned}
$$

Now, we consider the replacement $u \leftrightarrow w_{0}$ and $u-\sum_{j=0}^{n} w_{j} \leftrightarrow w_{n+1}$. Then the right-hand side is replaced by

$$
\begin{aligned}
& \sum_{m=1}^{k} \sum_{\substack{i_{1}=\cdots=i_{m-1}=0, i_{m}+\cdots+i_{k}=n-1}} N_{k}\left(w_{i_{1}}, \ldots, w_{i_{m-1}}, w_{i_{m}+1}, w_{i_{m+1}}, \ldots, w_{i_{k}}\right) \\
& \quad=\sum_{i_{1}+\cdots+i_{k}=n} N_{k}\left(w_{i_{1}}, \ldots, w_{i_{k}}\right)=w_{n+1} .
\end{aligned}
$$

Since $u$ has the upper bound estimate $\|u\|_{S} \leq C_{1}\|f\|_{D}$ which is the same as for $w_{0}$ up to a constant, we infer inductively that the upper bound estimates for $u-\sum_{l=0}^{n} w_{l}$ and for $w_{n+1}$ are also the same up to a constant.

The convergence of $\sum_{n=0}^{\infty} w_{n}$ is nothing but the well-posedness result. In fact, conversely, the solution $u$ is defined by $\sum_{n=0}^{\infty} w_{n}$. We now give a slightly different proof. It is known that $I(n, k)$, defined by (3.5), is the generalized Catalan number, and that $I(n, k)=$ $(n k) ! /(n(k-1)+1) ! n !$ (see [9]). Therefore, we conclude that $I(n, k) \sim\left(k^{k} /(k-1)^{k-1}\right)^{n}$ as $n \rightarrow \infty$. 
When we try to apply this theorem to the Cauchy problem of the nonlinear Schrödinger equation, we need the assumption that $\alpha$ is an even number. Indeed, (1.1) with (1.2) can be written as

$$
u(t)=U(t) \phi-i \int_{0}^{t} U(t-s)\left(|u|^{\alpha} u\right)(s) d s .
$$

Moreover, if the scattered state $\phi_{+}:=\lim _{t \rightarrow \infty} U(-t) u(t)$ exists then this equation is also written as

$$
u(t)=U(t) \phi_{+}+i \int_{t}^{\infty} U(t-s)\left(|u|^{\alpha} u\right)(s) d s .
$$

If $\alpha$ is an even integer then $|u|^{\alpha} u=u^{\alpha / 2+1} \bar{u}^{\alpha / 2}$ is $(\alpha+1)$-linear, and so this equation can be written as

$$
u=L(\phi)+N_{\alpha+1}(u, \ldots, u) .
$$

Then, setting suitable data space $D$ and solution space $S$, we can obtain the asymptotics of the solution from Theorem 3.2.

We want to extend this argument to the general case where $\alpha$ is not an even integer. However, if $\alpha$ is not an even integer, then the nonlinearity $|u|^{\alpha} u$ is no longer multilinear with respect to $u$. Hence, we make some modifications. Let $\lceil\alpha\rceil$ be the minimum integer larger than or equal to $\alpha$, and let $\beta:=\alpha /\lceil\alpha\rceil$. We split $|u|^{\alpha} u$ into $\left(|u|^{\beta}\right)^{\lceil\alpha\rceil} u$ and regard it as the product of one term of $u$ and $\lceil\alpha\rceil$ terms of $|u|^{\beta}$. Thus, the equation which we consider becomes the following type:

$$
u=L(\phi)+N_{\lceil\alpha\rceil+1}\left(|u|^{\beta}, \ldots,|u|^{\beta}, u\right) .
$$

It is important that $\beta \leq 1$, because we use the following estimate: if $0<b \leq 1$, then $\left|z_{1}\right|^{b}-\left|z_{2}\right|^{b} \leq\left|z_{1}-z_{2}\right|^{b}$, for all $z_{1}, z_{2} \in \mathbb{C}$. This inequality is the key for this modification.

Before treating (3.8), we consider the abstract equation

$$
u=L(f)+N_{k}\left(|u|^{b}, \ldots,|u|^{b}\right),
$$

where $k \geq 2,0<b \leq 1$, and the solution space $S$ is a space of complex-valued spacetime functions. Here, we take $k$ and $b$ independently. We note that (3.8) can be regarded as a mixture type of (3.1) and (3.9).

Assumption 3.3. Let $\left(D,\|\cdot\|_{D}\right)$ be a Banach space of initial data, and $\left(S,\|\cdot\|_{S}\right)$ be a Banach space of complex-valued spacetime functions. Moreover, the norm $\|\cdot\|_{S}$ is monotone, that is, if $|f| \leq|g|$ then $\|f\|_{S} \leq\|g\|_{S}$. The operator L satisfies

$$
\|L(f)\|_{S} \leq C_{0}\|f\|_{D}
$$

for some positive constant $C_{0}$. There exists a constant $\varepsilon$ such that

$$
\left\|N_{k}\left(u_{1}, \ldots, u_{k}\right)\right\|_{S} \leq \varepsilon \prod_{l=1}^{k}\left\|\left|u_{l}\right|^{1 / b}\right\|_{S}^{b} .
$$

THEOREM 3.4. Suppose that (3.9) satisfies Assumption 3.3 with the same b. Assume further that the constant $\varepsilon$ given by Assumption 3.3 is sufficiently small. Let $u$ be a solution of (3.9) with $\|u\|_{S} \leq C_{1}\|f\|_{D}$ with some constant $C_{1}$. If we define the approximate solution $w_{n}$ by 
the recursive formula

$$
w_{0}:=L(f), \quad w_{n}:=\sum_{\substack{i_{1}, \ldots, i_{k} \geq 0 \\ i_{1}+\cdots+i_{k}=n-1}} N_{k}\left(\widetilde{w}_{i_{1}}, \ldots, \widetilde{w}_{i_{k}}\right),
$$

where $\tilde{w}_{0}=\left|w_{0}\right|^{b}$ and $\widetilde{w}_{n}=\left|\sum_{l=0}^{n} w_{l}\right|^{b}-\left|\sum_{l=0}^{n-1} w_{l}\right|^{b}$, then we have the following boundedness and asymptotics:

$$
\begin{gathered}
\left\|w_{n}\right\|_{S} \leq I_{b}(n, k) \varepsilon^{e(n)}\left(C_{0}\|f\|_{D}\right)^{(k-1) e(n)} \\
\left\|u-\sum_{l=0}^{n} w_{l}\right\|_{S} \leq I_{b}(n+1, k) \varepsilon^{e(n+1)}\left(\left(C_{0} \vee C_{1}\right)\|f\|_{D}\right)^{(k-1) e(n+1)},
\end{gathered}
$$

where $e(n)=1+b+\cdots+b^{n-1}$ and $I_{b}(n, k)$ is defined by

$$
I_{b}(0, k)=1, \quad I_{b}(n, k)=\sum_{i_{1}+\cdots+i_{k}=n-1} \prod_{l=1}^{k}\left(I_{b}\left(i_{l}, k\right)\right)^{b} .
$$

Furthermore, if $b=1$ then $w_{n}$ gives the absolutely convergent expansion

$$
u=\sum_{n=0}^{\infty} w_{n} \quad \text { in } S .
$$

Proof. By the definition of $\widetilde{w}_{n}$, we deduce that

$$
\begin{gathered}
\left|\widetilde{w}_{n}\right| \leq\left.|| \sum_{l=0}^{n} w_{l}\right|^{b}-\left.\left|\sum_{l=0}^{n-1} w_{l}\right|^{b}|\leq| w_{n}\right|^{b}, \\
\left.|| u\right|^{b}-\sum_{l=0}^{n} \widetilde{w}_{l}|=||u|^{b}-\left|\sum_{l=0}^{n} w_{l}\right|^{b}|\leq| u-\left.\sum_{l=0}^{n} w_{l}\right|^{b},
\end{gathered}
$$

and so that

$$
\begin{gathered}
\left\|\widetilde{w}_{n}\right\|_{S} \leq\left\|w_{n}\right\|_{S}^{b}, \\
\left\|u-\sum_{l=0}^{n} \widetilde{w}_{l}\right\|_{S} \leq\left\|u-\sum_{l=0}^{n} w_{l}\right\|_{S}^{b}
\end{gathered}
$$

by the monotonicity of $\|\cdot\|_{S}$. Therefore, if (3.10) holds for $n=0,1, \ldots, n_{0}-1$ then the calculation similar to (3.6) shows that

$$
\begin{aligned}
\left\|w_{n_{0}}\right\|_{S} & =\left\|\sum_{i_{1}+\cdots+i_{k}=n_{0}-1} N_{k}\left(\widetilde{w}_{i_{1}}, \ldots, \widetilde{w}_{i_{k}}\right)\right\|_{S} \\
& \leq \varepsilon \sum_{i_{1}+\cdots+i_{k}=n_{0}-1} \prod_{m=1}^{k}\left(I_{b}\left(i_{m}, k\right) \varepsilon^{e\left(i_{m}\right)}\left(C_{0}\|f\|_{D}\right)^{(k-1) e\left(i_{m}\right)}\right)^{b} \\
& =I_{b}\left(n_{0}, k\right) \varepsilon^{e\left(n_{0}\right)}\left(C_{0}\|f\|_{D}\right)^{(k-1) e\left(n_{0}\right)},
\end{aligned}
$$

since $\sum_{m=1}^{k} b e\left(i_{m}\right) \geq b e\left(n_{0}-1\right)+(k-1) b e(0)=e\left(n_{0}\right)$. 
Since $N_{k}$ is $k$-linear, the same computation as (3.7) is possible, and so

$$
\begin{aligned}
u-\sum_{l=0}^{n} w_{l} & =N_{k}\left(|u|^{b}, \ldots,|u|^{b}\right)-\sum_{l=1}^{n} w_{l} \\
& =\sum_{m=1}^{k} \sum_{i_{m}+\cdots+i_{k}=n-1} N_{k}\left(|u|^{b}, \ldots,|u|^{b},|u|^{b}-\sum_{l=0}^{i_{m}} \widetilde{w}_{l}, \widetilde{w}_{i_{m+1}}, \ldots, \widetilde{w}_{i_{k}}\right) .
\end{aligned}
$$

Hence, by replacing $|u|^{b}$ and $|u|^{b}-\sum_{l=0}^{n} \widetilde{w}_{l}$ by $\widetilde{w}_{0}$ and $\widetilde{w}_{n+1}$, respectively, and using the estimate (3.13) instead of (3.14), we see that the upper bound estimates for $u-\sum_{l=0}^{n} w_{l}$ and $w_{n+1}$ is the same up to a constant. The convergence result is clear since $I_{b}(n, k)=I(n, k)$ and $e(n)=n$ if $b=1$.

Finally we treat the mixture-type equation (3.8). Let us consider the equation

$$
u=L(f)+N_{k+1}\left(|u|^{b}, \ldots,|u|^{b}, u\right) .
$$

We suppose that $k$ and $b$ are independent, which generalizes (3.8) slightly.

Assumption 3.5. We assume that $D, S$, and $L$ satisfy the same assumption as in Assumption 3.3. Moreover, there exists a constant $\varepsilon$ such that

$$
\left\|N_{k+1}\left(u_{1}, \ldots, u_{k+1}\right)\right\|_{S} \leq \varepsilon\left(\prod_{l=1}^{k}\left\|\left|u_{l}\right|^{1 / b}\right\|_{S}^{b}\right)\left\|u_{k+1}\right\|_{S} .
$$

THEOREM 3.6. Suppose that (3.17) satisfies Assumption 3.5 with the same b. Assume further that the constant $\varepsilon$ given by Assumption 3.5 is sufficiently small. Let $u$ be a solution of (3.17) with $\|u\|_{S} \leq C_{1}\|f\|_{D}$ with some constant $C_{1}$. If we define the approximate solution $w_{n}$ by the recursive formula

$$
w_{0}:=L(f), \quad w_{n}:=\sum_{\substack{i_{1}, \ldots, i_{k+1} \geq 0 \\ i_{1}+\cdots+i_{k+1}=n-1}} N_{k+1}\left(\widetilde{w}_{i_{1}}, \ldots, \widetilde{w}_{i_{k}}, w_{i_{k+1}}\right),
$$

then we have the following boundedness and asymptotics:

$$
\begin{gathered}
\left\|w_{n}\right\|_{S} \leq I_{b}^{\prime}(n, k+1) \varepsilon^{e(n)}\left(C_{0}\|f\|_{D}\right)^{k e(n)} \\
\left\|u-\sum_{l=0}^{n} w_{l}\right\|_{S} \leq I_{b}^{\prime}(n+1, k+1) \varepsilon^{e(n+1)}\left(\left(C_{0} \vee C_{1}\right)\|f\|_{D}\right)^{k e(n+1)},
\end{gathered}
$$

where $e(n)=1+b+\cdots+b^{n-1}$ and $I_{b}^{\prime}(n, k+1)$ is defined by

$$
\begin{gathered}
I_{b}^{\prime}(0, k+1)=1, \\
I_{b}^{\prime}(n, k+1)=\sum_{i_{1}+\cdots+i_{k+1}=n-1}\left(\prod_{l=1}^{k}\left(I_{b}^{\prime}\left(i_{l}, k+1\right)\right)^{b}\right) I_{b}^{\prime}\left(i_{k+1}, k+1\right) .
\end{gathered}
$$

Furthermore, if $b=1$, then $w_{n}$ gives the absolutely convergent expansion

$$
u=\sum_{n=0}^{\infty} w_{n} \text { in } S .
$$


Proof. It suffices to mention that

$$
\varepsilon^{e(n)}\left(C_{0}\|f\|_{D}\right)^{k e(n)} \leq 1 \quad \text { and } \quad \varepsilon^{e(n+1)}\left(\left(C_{0} \vee C_{1}\right)\|f\|_{D}\right)^{k e(n+1)} \leq 1
$$

if $\varepsilon$ is small. Then, using the inequality $x \leq x^{b}$ for $x \in[0,1]$ and $b \in(0,1]$, we can repeat the same computation as in Theorem 3.4.

Remark 3.1. There are several ways to split the nonlinearity $|u|^{\alpha} u$ into a product $u^{k_{1}} \bar{u}^{k_{2}}\left(\prod_{i}|u|^{b_{i}}\right)$ with $b_{i} \leq 1$ and $k_{1}, k_{2} \in \mathbb{N} \cup\{0\}$. However, the best way is $|u|^{\alpha} u=$ $\left(|u|^{\beta}\right)^{\lceil\alpha\rceil} u$ since the same argument as in the proof of Theorem 3.6 shows that the accuracy of the asymptotics depends on the infimum of $b_{i}$.

\section{Proofs of Theorems 1,2 , and 4}

In this section we apply the abstract result in the previous section and prove Theorem 1 and the some results in Theorem 4. Speaking in detail, Theorem 1 follows from Theorem 3.6, and the upper bound estimates and the convergence result in Theorem 4 follows from Theorem 3.2. Theorem 2 is shown directly (without the abstract result).

\subsection{The proof of Theorem 1}

Proof of Theorem 1. We first note that (1.7) is of the form (3.17). We set $D=X_{\rho, 1}$. All we have to do is to choose a suitable solution space $S$ so that Assumption 3.5 holds. We first let $S=Y^{2}(T) \cap Y^{\alpha+2}(T)$. It follows from (2.1) and (2.3) that

$$
\|G\|_{Y^{\alpha+2}(T)} \leq C T^{1-\theta+\delta(\alpha+2)}\left(\prod_{k=1}^{\lceil\alpha\rceil}\left\|\left|u_{k}\right|^{1 / \beta}\right\|_{Y^{\alpha+2}(T)}^{\beta}\right)\left\|u_{\lceil\alpha\rceil+1}\right\|_{Y^{\alpha+2}(T)}
$$

and

$$
\|G\|_{L^{\infty}\left(I ; L^{2}\right)} \leq C T^{1-\theta+\delta(\alpha+2) / 2}\left(\prod_{k=1}^{\lceil\alpha\rceil}\left\|\left|u_{k}\right|^{1 / \beta}\right\|_{Y^{\alpha+2}(T)}^{\beta}\right)\left\|u_{\lceil\alpha\rceil+1}\right\|_{Y^{\alpha+2}(T)},
$$

respectively. Therefore, we see from $Y^{2}(T)=L^{\infty}\left(I ; L^{2}\right)$ that

$$
\|G\|_{S} \leq C T^{1-\theta+\delta(\alpha+2)}\left(\prod_{k=1}^{\lceil\alpha\rceil}\left\|\left|u_{k}\right|^{1 / \beta}\right\|_{S}^{\beta}\right)\left\|u_{\lceil\alpha\rceil+1}\right\|_{S} .
$$

Then, applying Theorem 3.6 and using $Y^{2}(T) \cap Y^{\alpha+2}(T) \hookrightarrow Y^{r}(T)$ for $r \in[2, \alpha+2]$, we obtain the result for $r \in[2, \alpha+2]$.

Let us proceed to the case $r>\alpha+2$, we let $S$ be

$$
Z^{r}(T)=\bigcap_{(\alpha+1) r /(r-1) \leq p \leq r} Y^{p}(T) .
$$

We apply (2.1) with $s_{1}=\cdots=s_{\lceil\alpha\rceil+1}=(\alpha+1) r /(r-1)$ and $r=r$, and also with $s_{1}=$ $\cdots=s_{\lceil\alpha\rceil+1}=(\alpha+1)^{2} r /(\alpha r+1)$ and $r=(\alpha+1) r /(r-1)$. Then, we conclude that

$$
\|G\|_{S} \leq C T^{1-\theta+\delta(r)}\left(\prod_{k=1}^{\lceil\alpha\rceil}\left\|\left|u_{k}\right|^{1 / \beta}\right\|_{S}^{\beta}\right)\left\|u_{\lceil\alpha\rceil+1}\right\|_{S} .
$$

Therefore, Theorem 3.6 provides the result. The results for $L^{q_{0}}\left(I ; L^{r_{0}}\right)$ norm are immediate consequences of the results for $Y^{r}(T)$ norm by virtue of (2.3). 


\subsection{The upper bound estimates in Theorem 4}

Proof of Theorem 4. Equation (1.20) is of the form (3.1). We let $D=X_{\rho, 1}$ and $S=\Sigma^{\rho}(T)$. Lemma 2.3 tells us that Assumption 3.1 holds. Then, Theorem 3.2 shows that

$$
\left\|w_{n}\right\|_{\Sigma^{\rho}(T)} \leq C T^{n(1-\theta)}, \quad\left\|u-\sum_{k=0}^{n} w_{k}\right\|_{\Sigma^{\rho}(T)} \leq C T^{(n+1)(1-\theta)},
$$

and

$$
u=\sum_{n=0}^{\infty} w_{n} \quad \text { in } \Sigma^{\rho}(T) .
$$

By the embedding $\Sigma^{\rho}(T) \hookrightarrow Y^{r}(T)$, we see that these results also hold with $\|\cdot\|_{Y^{r}(T)}$ for all $r \in[2, \infty]$.

\subsection{The proof of Theorem 2}

We refine the estimate of $w_{n}$ and of $u-\sum_{k=0}^{n} w_{k}$ for $n=0,1,2$ by using the property $\mathcal{F} \phi_{+} \in H^{\rho}$. We prove the following proposition which contains Theorem 2 .

Proposition 4.1. Let $N \geq 1$ and $\alpha \in\left(\gamma(N), \alpha_{0}(N)\right)$. Put $\theta=\alpha N / 2$. Let $T \gg 1$ and $I=$ $[T, \infty)$. Let $\rho$ satisfy $1 \leq \rho<(\alpha+1) \wedge 2$. Then, it holds for all $r \in[2, \infty)$ with $\delta(r) \leq \rho$ $(r \in[2, \infty]$ if $\rho>N / 2)$ and admissible pair $\left(q_{0}, r_{0}\right)$ that

$$
\left\|w_{0}\right\|_{Y^{r}(T)} \leq C, \quad\left\|w_{0}\right\|_{L^{q_{0}\left(I ; L^{\left.q_{0}\right)}\right.}} \leq C, \quad\|u\|_{Y^{r}(T)} \leq C, \quad\|u\|_{L^{q_{0}\left(I ; L^{r}\right)}} \leq C .
$$

In the following, let $r \in[2, \infty]$ satisfy $\delta(r)<(\theta-1) \wedge 1$.

(1) Assume further that $N \leq 10$ and $N \alpha / 2(\alpha+1) \leq \rho$ then

$$
\begin{aligned}
\left\|w_{1}\right\|_{Y^{r}(T)} \leq C T^{1-\theta+\delta(r)}, & \left\|w_{1}\right\|_{L^{q_{0}}\left(I ; L^{\left.r_{0}\right)}\right.} \leq C T^{1-\theta}, \\
\left\|u-w_{0}\right\|_{Y^{r}(T)} \leq C T^{1-\theta+\delta(r)}, & \left\|u-w_{0}\right\|_{L^{q_{0}\left(I ; L^{r_{0}}\right)} \leq C T^{1-\theta} .}
\end{aligned}
$$

(2) Assume further that $N \leq 8$ and

$$
\frac{\theta(1-\beta)+\beta}{\alpha-\beta+1} \vee \frac{\theta-\beta}{\alpha-\beta+1}<\rho .
$$

Then,

$$
\begin{aligned}
& \left\|w_{2}\right\|_{Y^{r}(T)}=o\left(T^{1-\theta+\delta(r)}\right), \quad\left\|u-w_{0}-w_{1}\right\|_{Y^{r}(T)}=o\left(T^{1-\theta+\delta(r)}\right), \\
& \left\|w_{2}\right\|_{L^{q_{0}\left(I ; L^{\left.r_{0}\right)}\right.}}=o\left(T^{1-\theta}\right), \quad\left\|u-w_{0}-w_{1}\right\|_{L^{q_{0}\left(I ; L^{\left.r_{0}\right)}\right.}}=o\left(T^{1-\theta}\right) .
\end{aligned}
$$

(3) Assume further that $N \leq 5$ and

$$
\frac{N \alpha}{2(\alpha-\beta+1)} \vee\left(\frac{N}{2}-\frac{2(1-\beta)(\theta-1)}{\alpha}\right) \leq \rho
$$

$(\rho>N / 2$ if $\beta=1)$. Then,

$$
\begin{aligned}
& \left\|w_{2}\right\|_{Y^{r}(T)} \leq C T^{(1-\theta)(1+\beta)+\delta(r)}, \\
& \left\|u-w_{0}-w_{1}\right\|_{Y^{r}(T)} \leq C T^{(1-\theta)(1+\beta)+\delta(r)}, \\
& \left\|w_{2}\right\|_{L^{q_{0}}\left(I ; L^{\left.r_{0}\right)}\right.} \leq C T^{(1-\theta)(1+\beta)}, \\
& \left\|u-w_{0}-w_{1}\right\|_{L^{q_{0}}\left(I ; L^{\left.r_{0}\right)}\right.} \leq C T^{(1-\theta)(1+\beta)} .
\end{aligned}
$$


Moreover, if $\alpha>1$ then

$$
\left\|u-w_{0}-w_{1}-w_{2}\right\|_{L^{q_{0}\left(I ; L^{r}\right)}}=o\left(T^{(1-\theta)(1+\beta)}\right) .
$$

Remark 4.1. The restriction on the dimension of $N$ comes from the existence of $\rho$. By the existence result, $\rho$ must be smaller than $(\alpha+1) \wedge 2$. Then, the condition $N \alpha /(\alpha+1)<$ $(\alpha+1) \wedge 2$ holds in the following cases: $\alpha \in\left(\gamma(N), \alpha_{0}(N)\right)$ if $N \leq 8$ and $\alpha \in(\gamma(N),(N-$ $4-\sqrt{N(N-8)}) / 4$ ) if $N=9,10$. On the other hand, the left-hand side of (4.1) is bounded by $(\alpha+1) \wedge 2$ in the cases $\alpha \in\left(\gamma(N), \alpha_{0}(N)\right)$ if $N \leq 6$ and $\alpha \in(\gamma(N), 2 /(N-4))$ if $N=$ 7, 8, and the left-hand side of (4.2) is bounded by $(\alpha+1) \wedge 2$ in the cases $\alpha \in\left(\gamma(N), \alpha_{0}(N)\right)$ if $N \leq 3, \alpha \in\left(\gamma(4), \alpha_{0}(4)\right)$ and $\alpha \neq 1$ if $N=4$, and $\alpha \in\left(1, \alpha_{0}(5)\right)$ if $N=5$.

Remark 4.2. If $\alpha \in\left(\gamma(N), \alpha_{0}(N)\right)$ satisfies $\alpha>1$, then it holds that

$$
\frac{\theta(1-\beta)+\beta}{\alpha-\beta+1} \leq \frac{\theta-\beta}{\alpha-\beta+1} \vee 1, \quad \frac{N}{2}-\frac{2(1-\beta)(\theta-1)}{\alpha} \leq \frac{N \alpha}{2(\alpha-\beta+1)} .
$$

Proof of Proposition 4.1. By the Sobolev embedding, for $r \in[2, \infty)$ with $\delta(r) \leq \rho(r \in$ $[2, \infty]$ if $\rho>N / 2$ ), there exists a constant $C_{0}$ such that

$$
\begin{aligned}
\|u\|_{Y^{r}(T)} & \leq\|u\|_{\Sigma^{\rho}(T)} \leq C_{0}, \\
\left\|w_{0}\right\|_{Y^{r}(T)} & \leq\left\|w_{0}\right\|_{\Sigma^{\rho}(T)} \leq C_{0} .
\end{aligned}
$$

Step 1. We first prove the bounds of $u-w_{0}$ and $w_{1}$. Let us assume that $N \alpha / 2(\alpha+1)<\rho$. We deduce from (2.1), (4.3), and (4.4) that

$$
\begin{aligned}
\left\|u-w_{0}\right\|_{Y^{r}(T)}=\left\|G\left(|u|^{\beta}, \ldots,|u|^{\beta}, u\right)\right\|_{Y^{r}(T)} & \leq C T^{1-\theta+\delta(r)}\|u\|_{Y^{(\alpha+1) r^{\prime}}(T)}^{\alpha+1} \\
& \leq C T^{1-\theta+\delta(r)}
\end{aligned}
$$

and

$$
\begin{aligned}
\left\|w_{1}\right\|_{Y^{r}(T)}=\left\|G\left(\widetilde{w}_{0}, \ldots, \widetilde{w}_{0}, w_{0}\right)\right\|_{Y^{r}(T)} & \leq C T^{1-\theta+\delta\left(r_{1}\right)}\left\|w_{0}\right\|_{Y^{(\alpha+1) r^{\prime}}(T)}^{\alpha+1} \\
& \leq C T^{1-\theta+\delta(r)}
\end{aligned}
$$

if $\delta\left((\alpha+1) r^{\prime}\right)=(\theta-\delta(r)) /(\alpha+1) \leq \rho$. Therefore, if $N \alpha / 2(\alpha+1) \leq \rho$ then (4.5) and (4.6) hold for all $r$ with $0 \leq \delta(r)<1 \wedge(\theta-1)$. Similarly, we see from (2.3), (4.3), and (4.4) that

$$
\begin{gathered}
\left\|u-w_{0}\right\|_{L^{q_{0}}\left(I ; L^{r_{0}}\right)} \leq C T^{1-\theta}, \\
\left\|w_{1}\right\|_{L^{q_{0}\left(I ; L^{r_{0}}\right)} \leq C T^{1-\theta}}
\end{gathered}
$$

for all admissible pairs $\left(q_{0}, r_{0}\right)$.

Step 2. We proceed to the proof of the bounds of $u-w_{0}-w_{1}$ and $w_{2}$. Let us prove the second part of the proposition. Assume (4.1). An elementary calculation shows that

$$
\begin{aligned}
\| u & -w_{0}-w_{1} \|_{Y^{r}(T)} \\
& \leq\left\|G\left(|u|^{\beta}, \ldots,|u|^{\beta}, u-w_{0}\right)\right\|_{Y^{r}(T)} \\
& +\left\|\sum_{\substack{1 \leq l \leq\lceil\alpha\rceil \\
i_{1}=\cdots=i_{\lceil\alpha\rceil+1}=0}} G\left(|u|^{\beta}, \ldots,|u|^{\beta},|u|^{\beta}-\widetilde{w}_{i_{l}}, \widetilde{w}_{i_{l+1}}, \ldots, \widetilde{w}_{i_{\lceil\alpha]}}, w_{0}\right)\right\|_{Y^{r}(T)} \\
= & :\left\|\mathcal{I}_{1}\right\|_{Y^{r}(T)}+\left\|\mathcal{I}_{2}\right\|_{Y^{r}(T)} .
\end{aligned}
$$


We first estimate $\mathcal{I}_{2}$. We deduce from (2.1), (4.5), (4.3), and (4.4) that

$$
\begin{aligned}
\left\|\mathcal{I}_{2}\right\|_{Y^{r}(T)} & \leq C T^{1-\theta+\delta(r)} \sum_{l=1}^{\lceil\alpha\rceil}\left\|u-w_{0}\right\|_{Y^{s_{1}}(T)}^{\beta}\|u\|_{Y^{s_{2}(T)}}^{\beta(l-1)}\left\|w_{0}\right\|_{Y^{s_{2}(T)}}^{\alpha-\beta l+1} \\
& \leq C T^{1-\theta+\beta\left(1-\theta+\delta\left(s_{1}\right)\right)+\delta(r)}
\end{aligned}
$$

if $\delta\left(s_{1}\right)<(\theta-1) \wedge 1$ and $\delta\left(s_{2}\right)=\left(\delta(r)-\beta \delta\left(s_{1}\right)\right) /(\alpha-\beta+1) \leq \rho$. Now, we let $\delta\left(s_{1}\right)=$ $(\theta-1) \wedge 1-\varepsilon$ with $\varepsilon>0$. Then, we have

$$
\left\|\mathcal{I}_{2}\right\|_{Y^{r}(T)}=o\left(T^{1-\theta+\delta(r)}\right)
$$

and

$$
\delta\left(s_{2}\right)=\left(\frac{\theta(1-\beta)+\beta}{\alpha-\beta+1} \vee \frac{\theta-\beta}{\alpha-\beta+1}\right)+\frac{-\delta(r)+\beta \varepsilon}{\alpha-\beta+1} .
$$

If we take $\varepsilon$ to be small then the condition $\delta\left(s_{2}\right) \leq \rho$ holds for $r=2$ by assumption, and therefore it holds for all $r \geq 2$ with a suitable $\varepsilon$. Similarly, (2.3) provides that

$$
\left\|\mathcal{I}_{2}\right\|_{L^{q_{0}\left(I ; L^{\left.r_{0}\right)}\right.}}=o\left(T^{1-\theta}\right) .
$$

We next estimate $\mathcal{I}_{1}$. Take a small constant $\varepsilon>0$ and let $r_{1}$ satisfy $\delta\left(r_{1}\right)=1 \wedge(\theta-1)-\varepsilon$. Then, it follows that

$$
\begin{aligned}
\left\|\mathcal{I}_{1}\right\|_{Y^{r_{1}}(T)} & \leq C T^{1-\theta+\delta\left(r_{1}\right)}\|u\|_{Y^{s_{3}}(T)}^{\alpha}\left\|u-w_{0}\right\|_{Y^{s_{4}}(T)} \\
& \leq C T^{2(1-\theta)+\delta\left(r_{1}\right)+\delta\left(s_{4}\right)}\|u\|_{Y^{s_{3}}(T)}^{\alpha} .
\end{aligned}
$$

We take $s_{4}=r_{1}$. Then,

$$
\delta\left(s_{3}\right)=\frac{\theta-2((\theta-1) \vee 1)}{\alpha}+2 \varepsilon=\left|\frac{N}{2}-\frac{2}{\alpha}\right|+2 \varepsilon .
$$

We deduce that the right-hand side is bounded by one if $\varepsilon$ is small, so that $\|u\|_{Y^{s_{3}}(T)}$ is bounded. Then, (4.12) becomes

$$
\left\|\mathcal{I}_{1}\right\|_{Y^{r_{1}(T)}}=o\left(T^{1-\theta+\delta\left(r_{1}\right)}\right) .
$$

Moreover, it follows from (2.4) that

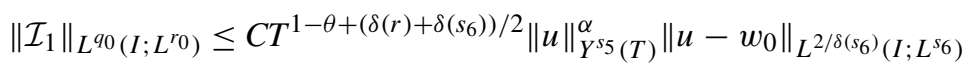

$$
\begin{aligned}
& \leq C T^{2(1-\theta)+\left(\delta(r)+\delta\left(s_{6}\right)\right) / 2}\|u\|_{Y^{s_{5}(T)}}^{\alpha} .
\end{aligned}
$$

We put $\delta(r)=\delta\left(s_{6}\right)=1 \wedge(\theta-1)-\varepsilon$. Then, $\delta\left(s_{5}\right)=\delta\left(s_{3}\right)$ and

$$
\left\|\mathcal{I}_{1}\right\|_{L^{q_{0}\left(I ; L^{\left.r_{0}\right)}\right.}}=o\left(T^{1-\theta}\right) \text {. }
$$

Interpolating (4.13) and this with $\left(q_{0}, r_{0}\right)=(\infty, 2)$, we obtain

$$
\left\|\mathcal{I}_{1}\right\|_{Y^{r}(T)}=o\left(T^{1-\theta+\delta(r)}\right)
$$

for all $2 \leq r \leq r_{1}$. Hence, from (4.16), (4.10), (4.15), and (4.11), we finally obtain

$$
\begin{gathered}
\left\|u-w_{0}-w_{1}\right\|_{Y^{r}(T)}=o\left(T^{1-\theta+\delta(r)}\right), \\
\left\|u-w_{0}-w_{1}\right\|_{L^{q_{0}}\left(I ; L^{r_{0}}\right)}=o\left(T^{1-\theta}\right),
\end{gathered}
$$


for all $r$ with $0 \leq \delta(r)<1 \wedge(\theta-1)$ and all admissible pairs $\left(q_{0}, r_{0}\right)$. The estimate of $w_{2}$ is performed in the same way. Only replacing $u$ and $u-w_{0}$ by $w_{0}$ and $w_{1}$ in the above estimation of $u-w_{0}-w_{1}$, respectively, we obtain

$$
\begin{gathered}
\left\|w_{2}\right\|_{Y^{r}(T)}=o\left(T^{1-\theta+\delta(r)}\right), \\
\left\|w_{2}\right\|_{L^{q_{0}}\left(I ; L^{r_{0}}\right)}=o\left(T^{1-\theta}\right)
\end{gathered}
$$

for all $r$ with $0 \leq \delta(r)<1 \wedge(\theta-1)$ and all admissible pairs $\left(q_{0}, r_{0}\right)$.

Step 3. In this and the next step, we prove the third part. Assume further that (4.2) holds. We refine the estimates in the previous step. We first consider $\mathcal{I}_{2}$. We take $s_{1}=2$. Then, (4.10) becomes

$$
\left\|\mathcal{I}_{2}\right\|_{Y^{r}(T)} \leq C T^{(1-\theta)(1+\beta)+\delta(r)}
$$

and $\delta\left(s_{2}\right)=(\theta-\delta(r)) /(\alpha-\beta+1)$. We note that $\delta\left(s_{2}\right) \leq \rho$ holds for $r=2$ by assumption, and therefore for all $r \geq 2$. In a similar way, we obtain

$$
\left\|\mathcal{I}_{2}\right\|_{L^{q_{0}\left(I ; L^{r} 0\right)}} \leq C T^{(1-\theta)(1+\beta)} .
$$

We next estimate $\mathcal{I}_{1}$. In (4.12), we take $s_{4}$ so that $\delta\left(s_{4}\right)=(\theta-1)(1-\beta)$. It is possible if $0 \leq(\theta-1)(1-\beta)<1 \wedge(\theta-1)$. We note that $\theta \geq 1$ if and only if $\alpha \geq 2 / N$, and that $0<$ $\beta \leq 1$ always holds. Therefore, $0 \leq(\theta-1)(1-\beta)<\theta-1$. Moreover, $(\theta-1)(1-\beta)<1$ is equivalent to $N \alpha^{2}-(N\lceil\alpha\rceil+2) \alpha+4\lceil\alpha\rceil>0$, which is easy to verify. Moreover, we take $\varepsilon$ so small that $\delta\left(r_{1}\right)>\delta\left(s_{4}\right)$. Then, it holds that

$$
\delta\left(s_{3}\right)=\frac{\theta-\delta\left(r_{1}\right)-\delta\left(s_{4}\right)}{\alpha}<\frac{\theta-2 \delta\left(s_{4}\right)}{\alpha}=\frac{N}{2}-\frac{2(\theta-1)(1-\beta)}{\alpha} .
$$

By assumption, we deduce that the right-hand side is bounded by $\rho$, so that $\|u\|_{Y^{s_{3}(T)}}$ is bounded. Thus, (4.12) becomes

$$
\left\|\mathcal{I}_{1}\right\|_{Y^{r_{1}(T)}} \leq C T^{(1-\theta)(1+\beta)+\delta\left(r_{1}\right)} .
$$

We next consider (4.14). Set $\delta(r)=\delta\left(s_{6}\right)=(\theta-1)(1-\beta)<1 \wedge(\theta-1)$. Then, $\delta\left(s_{5}\right)=$ $N / 2-2(\theta-1)(1-\beta) / \alpha \leq \rho$ by assumption and (4.14) becomes

$$
\left\|\mathcal{I}_{1}\right\|_{L^{q_{0}\left(I ; L^{r} 0\right)}} \leq C T^{(1-\theta)(1+\beta)} .
$$

Interpolating (4.23) and this with $\left(q_{0}, r_{0}\right)=(\infty, 2)$, we obtain

$$
\left\|\mathcal{I}_{1}\right\|_{Y^{r}(T)} \leq C T^{(1-\theta)(1+\beta)+\delta(r)}
$$

for all $2 \leq r \leq r_{1}$. Hence, from (4.25), (4.21), (4.24), and (4.22), we finally obtain

$$
\begin{gathered}
\left\|u-w_{0}-w_{1}\right\|_{Y^{r}(T)} \leq C T^{(1-\theta)(1+\beta)}, \\
\left\|u-w_{0}-w_{1}\right\|_{L^{q_{0}\left(I ; L^{r} 0\right)}} \leq C T^{(1-\theta)(1+\beta)}
\end{gathered}
$$

for all $r$ with $0 \leq \delta(r)<1 \wedge(\theta-1)$ and all admissible pairs $\left(q_{0}, r_{0}\right)$. The estimate of $w_{2}$ is performed in the same way. We obtain

$$
\begin{gathered}
\left\|w_{2}\right\|_{Y^{r}(T)} \leq C T^{(1-\theta)(1+\beta)}, \\
\left\|w_{2}\right\|_{L^{q_{0}\left(I ; L^{r_{0}}\right)}} \leq C T^{(1-\theta)(1+\beta)}
\end{gathered}
$$

for all $r$ with $0 \leq \delta(r)<1 \wedge(\theta-1)$ and all admissible pairs $\left(q_{0}, r_{0}\right)$. 
Step 4. The estimate of $u-w_{0}-w_{1}-w_{2}$. From the existence result (Proposition 1.1), $\rho$ must be smaller than $(\alpha+1) \wedge 2$. If $\lceil\alpha\rceil=1$, then the condition $N / 2-$ $2(1-\beta)(\theta-1) / \alpha<\alpha+1$ is equivalent to $2(N-1) \alpha^{2}-(N+6) \alpha+4<0$, which does not hold for all $\alpha \in\left(\gamma(N), \alpha_{0}(N)\right)$ with $\alpha \leq 1$ if $N \geq 5$. It means that we cannot take $\rho$ so that (4.2) is satisfied if $\alpha \leq 1$ and $N \geq 5$. Hence, in the following, we only consider $N=4$ when we are concerned with the case $\alpha \leq 1$.

We estimate $w_{3}$ instead of $u-w_{0}-w_{1}-w_{2}$ because these two estimates are shown in the same way according to the replacements $|u|^{b} \leftrightarrow \widetilde{w}_{0},|u|^{b}-\sum_{k=0}^{n-1} \widetilde{w}_{k} \leftrightarrow \widetilde{w}_{n}(n \geq 1)$, $u \leftrightarrow w_{0}$, and $u-\sum_{k=0}^{n-1} w_{k} \leftrightarrow w_{n}(n \geq 1)$. Now, $w_{3}$ is equal to

$$
\sum_{i_{1}+\cdots+i_{\lceil\alpha\rceil+1}=2} G\left(\widetilde{w}_{i_{1}}, \ldots, \widetilde{w}_{\lceil\alpha\rceil}, w_{\lceil\alpha\rceil+1}\right) .
$$

There exist four cases:

(1) one of $i_{1}, \ldots, i_{\lceil\alpha\rceil}$ is two and the others are zero;

(2) $i_{\lceil\alpha\rceil+1}$ is two and the others are zero;

(3) two of $i_{1}, \ldots, i_{\lceil\alpha\rceil}$ are one and the others are zero;

(4) $i_{\lceil\alpha\rceil+1}$ is one and one of $i_{1}, \ldots, i_{\lceil\alpha\rceil}$ is one, the others are zero.

We write $w_{3}=$ I + II + III + IV, where I, II, III, and IV are the sums of the terms which belong to the first, second, third, and fourth case, respectively. The third case occurs only if $\lceil\alpha\rceil \geq 2$. Therefore, we let III be identically zero if $\alpha \leq 1$. We deduce from (2.3), (4.4), and (4.28) that

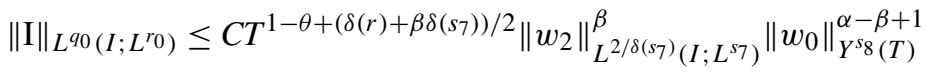

$$
\begin{aligned}
& \leq C T^{(1-\theta)\left(1+\beta+\beta^{2}\right)+\left(\delta(r)+\beta \delta\left(s_{7}\right)\right) / 2} .
\end{aligned}
$$

We set $\delta(r)=\delta\left(s_{7}\right)=\left(\left(2(\theta-1) \beta^{2} /(1+\beta)\right) \wedge(\theta /(1+\beta)) \wedge 1\right)-\varepsilon$ with a small positive constant $\varepsilon$, which gives us

$$
\|\mathrm{I}\|_{L^{q_{0}\left(I ; L^{r_{0}}\right)}}=o\left(T^{(1-\theta)(1+\beta)}\right) .
$$

This choice is possible if $\left(\delta(r)+\beta \delta\left(s_{7}\right)\right) / 2<\theta-1$ and $\delta\left(s_{8}\right)=\left(\theta-\delta(r)-\beta \delta\left(s_{7}\right)\right) /(\alpha-$ $\beta+1) \leq \rho$. The first condition is always satisfied because $\left(\delta(r)+\beta \delta\left(s_{7}\right)\right) / 2<\beta^{2}(\theta-1) \leq$ $(\theta-1)$ by definition. Taking $\varepsilon$ small, one sees that the second condition holds if

$$
f_{1}(\alpha) \vee f_{2}(\alpha):=\frac{\theta-\beta-1}{\alpha-\beta+1} \vee \frac{\theta-2 \beta^{2}(\theta-1)}{\alpha-\beta+1}<\rho .
$$

Similarly,

$$
\begin{aligned}
&\|\mathrm{II}\|_{L^{q_{0}\left(I ; L^{\left.r_{0}\right)}\right.}} \leq C T^{1-\theta+\left(\delta(r)+\delta\left(s_{9}\right)\right) / 2}\left\|w_{2}\right\|_{L^{2 / \delta\left(s_{9}\right)}\left(I ; L^{\left.s_{9}\right)}\right.}\|u\|_{Y^{s_{10}(T)}}^{\alpha} \\
& \leq C T^{(1-\theta)(2+\beta)+\left(\delta(r)+\delta\left(s_{9}\right)\right) / 2} .
\end{aligned}
$$

If we choose $\delta(r)=\delta\left(s_{9}\right)=1 \wedge(\theta-1)-\varepsilon$, then we obtain

$$
\|\mathrm{II}\|_{L^{q_{0}\left(I ; L^{\left.r_{0}\right)}\right.}}=o\left(T^{(1-\theta)(1+\beta)}\right) .
$$

The above choice is possible if $\delta\left(s_{10}\right) \leq \rho$, that is, if $|N / 2-2 / \alpha|<\rho$. We note that $|N / 2-2 / \alpha|<1$ holds for all $\alpha \in\left(\gamma(N), \alpha_{0}(N)\right)$. We next estimate III. Without any loss 
of generality, we can assume that $i_{1}=i_{2}=1$ and $i_{3}=\cdots=i_{\lceil\alpha\rceil+1}=0$. It follows from (2.4) with $K=\{1,2\}$, (4.4), (4.8) that

$$
\begin{aligned}
\|\mathrm{III}\|_{L^{q_{0}\left(I ; L^{r} 0\right)}} \leq C T^{1-\theta+\left(\delta(r)+2 \beta \delta\left(s_{11}\right)\right) / 2}\left\|w_{1}\right\|_{L^{2 / \delta\left(s_{11}\right)}\left(I ; L^{\left.s_{11}\right)}\right.}^{2 \beta}\left\|w_{0}\right\|_{Y^{s_{12}(T)}}^{\alpha-2 \beta+1} \\
\leq C T^{(1-\theta)(1+2 \beta)+\left(\delta(r)+2 \beta \delta\left(s_{11}\right)\right) / 2} .
\end{aligned}
$$

Choosing $\delta(r)=\delta\left(s_{11}\right)=((\theta-1) 2 \beta /(2 \beta+1)) \wedge 1-\varepsilon$, we obtain

$$
\|\mathrm{IIII}\|_{L^{q_{0}\left(I ; L^{\left.r_{0}\right)}\right.}}=o\left(T^{(1-\theta)(1+\beta)}\right) .
$$

This choice is possible if $\delta\left(s_{12}\right) \leq \rho$ and $\left(\delta(r)+2 \beta \delta\left(s_{11}\right)\right) / 2<\theta-1$, which follows from the condition

$$
f_{3}(\alpha) \vee f_{4}(\alpha):=\frac{2 \beta-(2 \beta-1) \theta}{\alpha-2 \beta+1} \vee \frac{\theta-2 \beta-1}{\alpha-2 \beta+1}<\rho
$$

and the definition of $\delta(r)$ and $\delta\left(s_{11}\right)$, respectively. We turn to the estimate of IV. We consider the case where $i_{1}=i_{\lceil\alpha\rceil+1}=1$ and the others are zero. Then, applying (2.5), we obtain

$$
\begin{aligned}
\|\mathrm{IV}\|_{L^{q_{0}}\left(I ; L^{\left.r_{0}\right)}\right.} & \leq C T^{1-\theta+\left(\delta(r)+(\beta+1) \delta\left(s_{13}\right)\right) / 2}\left\|w_{1}\right\|_{L^{2 / \delta\left(s_{13}\right)}\left(I ; L^{\left.s_{13}\right)}\right.}^{\beta+1}\left\|w_{0}\right\|_{Y^{s_{14}}(T)}^{\alpha-\beta} \\
& \leq C T^{(1-\theta)(2+\beta)+\left(\delta(r)+(\beta+1) \delta\left(s_{13}\right)\right) / 2} .
\end{aligned}
$$

We first consider the case $\lceil\alpha\rceil \geq 2$. Then, we take the above powers so that $\delta(r)=\delta\left(s_{13}\right)=$ $2(\theta-1) /(\beta+2) \wedge 1-\varepsilon$ and obtain

$$
\|\mathrm{IV}\|_{L^{q_{0}}\left(I ; L^{r_{0}}\right)} \leq o\left(T^{(1-\theta)(1+\beta)}\right) .
$$

This choice is admissible if

$$
f_{5}(\alpha) \vee f_{6}(\alpha):=\frac{2-\theta}{\alpha-\beta} \vee \frac{\theta-\beta-2}{\alpha-\beta}<\rho .
$$

We now assume $\lceil\alpha\rceil=1$. Then,

$$
\begin{aligned}
&\|\mathrm{IV}\|_{L^{q_{0}\left(I ; L^{\left.r_{0}\right)}\right.}} \leq C T^{(1-\theta)(2+\alpha)+\left(\delta(r)+(\alpha+1) \delta\left(s_{13}\right)\right) / 2} \\
&=C T^{(1-\theta)(1+\alpha)+1-\theta / 2}
\end{aligned}
$$

since the powers $r$ and $s_{13}$ satisfy $\delta(r)+(\alpha+1) \delta\left(s_{13}\right)=\theta$. Therefore, we obtain (4.39) if $\theta>2$, which is equivalent to $\alpha>4 / N$. Since $N=4$ and $\alpha \leq 1$, it is impossible. Summarizing (4.31), (4.34), (4.36), and (4.39), we finally obtain

$$
\left\|w_{3}\right\|_{L^{q_{0}\left(I ; L^{\left.r_{0}\right)}\right.}}=o\left(T^{(1-\theta)(1+\beta)}\right) .
$$

The condition is $1 \leq N \leq 5, \gamma(N) \vee 1<\alpha<\alpha_{0}(N)$, and $\max _{1 \leq i \leq 6} f_{i}(\alpha)<\rho$. We claim that these conditions can be summarized as $\rho \geq 1$ if $1 \leq N \leq 4$, and $\alpha>1, \rho \geq 1$, and $\rho>f_{2}(\alpha)$ if $N=5$. Let us first note that $\max _{1 \leq i \leq 6} f_{i}(\alpha)<N / 2$ always holds. This is because $\delta(r)<$ $N / 2$ holds if $r<\infty$ in general. Here, by the definitions of $s_{8}, s_{12}$, and $s_{14}$, they are finite. Hence, $\max _{1 \leq i \leq 6} f_{i}(\alpha)<N / 2$. This implies that the condition $\rho \geq 1$ is sufficient if $N=1,2$. We next consider $N=3$. Here $\gamma(3)=1$ and $\alpha_{0}(3)=4$. Regarding $\lceil\alpha\rceil$ as a constant, we consider that $f_{i}(\alpha)$ is a function only of $\alpha$ in the interval $(\lceil\alpha\rceil-1,\lceil\alpha\rceil)$ for $i=1, \ldots, 6$. Then, by calculating directly, we verify for $\lceil\alpha\rceil=2,3,4$ that the functions $f_{1}, f_{4}, f_{6}$ are 
strictly increasing and the functions $f_{2}, f_{3}, f_{5}$ are strictly decreasing. Moreover, it holds that $\lim _{\alpha \uparrow\lceil\alpha\rceil} f_{i}(\alpha) \leq 1(i=1,4,6)$ and $\lim _{\alpha \downarrow\lceil\alpha\rceil-1} f_{i}(\alpha) \leq 1(i=2,3,5)$. The equal signs in the first limit and the second limit can hold only if $\lceil\alpha\rceil=4$ and $\lceil\alpha\rceil=2$, respectively. Therefore, we deduce that $\max _{1 \leq i \leq 6} f_{i}(\alpha)<1$. The case $N=4$ is handled in the same way. If $N=5$, then the functions $f_{1}, f_{4}, f_{6}$ are strictly increasing and the functions $f_{2}, f_{3}, f_{5}$ are strictly decreasing. Moreover, it holds that $\lim _{\alpha \uparrow 2} f_{i}(\alpha) \leq 1(i=1,4,6), \lim _{\alpha \downarrow 1} f_{i}(\alpha) \leq 1$ $(i=3,5)$, and $\lim _{\alpha \downarrow 1} f_{2}(\alpha)=7 / 6>1$. Therefore, we need the condition $f_{2}(\alpha)<\rho$ and $\rho \geq 1$ in this case. However, the condition $f_{2}(\alpha)<\rho$ is weaker than (4.2) under $\rho \geq 1$. This can be seen by using a similar argument. In the end, we conclude that (4.41) holds if (4.2) holds and $\alpha>1$. As stated above, the same proof gives us that

$$
\left\|u-w_{0}-w_{1}-w_{2}\right\|_{L^{q_{0}\left(I ; L^{r_{0}}\right)}}=o\left(T^{(1-\theta)(1+\beta)}\right),
$$

which completes the proof of Proposition 4.1, and so of Theorem 2.

\section{The derivation of $V_{n}$}

In the rest of the paper, we consider the approximation of $w_{n}$. In this section, we first discuss formally the approximation of $w_{n}$, and we see where $V_{n}$ comes from. The rigorous proofs are in Section 6.

We first consider the case $\alpha$ as an even number. Let $w_{n}$ be defined by (1.21). We begin with the approximation of $w_{0}=U(t) \phi_{+}$. By the factorization of $U(t)$, it is written as

$$
M(t) D(t) \mathcal{F} M(t) \phi_{+}=M(t) D(t)\left(\mathcal{F} M(t) \mathcal{F}^{-1}\right) \mathcal{F} \phi_{+}
$$

The operator $\mathcal{F} M(t) \mathcal{F}^{-1}$ is equal to $e^{-i(\Delta / 4 t)}=U(-1 / 4 t)$. Now, let us regard this operator as the identity Id since we are dealing with very large $t$. Then, we obtain $V_{0}(t)=$ $M(t) D(t) \mathcal{F} \phi_{+}$. Let us proceed to the approximation of $w_{1}$. Since

$$
\begin{aligned}
w_{1}(t) & =H\left(w_{0}, \ldots, w_{0}\right)=i \int_{t}^{\infty} U(t-s)\left|w_{0}(s)\right|^{\alpha} w_{0}(s) d s \\
& =i M(t) D(t) \int_{t}^{\infty}\left(\mathcal{F} M(t) M(s)^{-1} \mathcal{F}^{-1}\right) D(s)^{-1} M(s)^{-1}\left|w_{0}(s)\right|^{\alpha} w_{0}(s) d s,
\end{aligned}
$$

replacing $w_{0}$ and the operator $\mathcal{F} M(t) M(s)^{-1} \mathcal{F}^{-1}=U(1 / 4 s-1 / 4 t)$ with $V_{0}$ and Id, respectively, we obtain

$$
\begin{aligned}
V_{1} & =i M(t) D(t) \int_{t}^{\infty} D(s)^{-1} M(s)^{-1}\left|V_{0}(s)\right|^{\alpha} V_{0}(s) d s \\
& =i M(t) D(t) \int_{t}^{\infty}|2 s|^{-\theta}\left|\mathcal{F} \phi_{+}\right|^{\alpha} \mathcal{F} \phi_{+} \\
& =i \frac{t^{1-\theta}}{2^{\theta}(\theta-1)} M(t) D(t)\left|\mathcal{F} \phi_{+}\right|^{\alpha} \mathcal{F} \phi_{+} .
\end{aligned}
$$


By a similar replacement, the function $w_{2}$, which is written as

$$
\begin{aligned}
w_{2}(t)= & \sum_{i_{1}+\cdots+i_{\alpha+1}=1} H\left(w_{i_{1}}, \ldots, w_{i_{\alpha+1}}\right) \\
= & i \frac{\alpha+2}{2} \int_{t}^{\infty} U(t-s)\left|w_{0}(s)\right|^{\alpha} w_{1}(s) d s \\
& +i \frac{\alpha}{2} \int_{t}^{\infty} U(t-s)\left|w_{0}(s)\right|^{\alpha-2}\left(w_{0}(s)\right)^{2} \overline{w_{1}(s)} d s,
\end{aligned}
$$

will be approximated by

$$
\begin{aligned}
& i \frac{\alpha+2}{2} M(t) D(t) \int_{t}^{\infty} D(s)^{-1} M(s)^{-1}\left|V_{0}(s)\right|^{\alpha} V_{1}(s) d s \\
& \quad+i \frac{\alpha}{2} M(t) D(t) \int_{t}^{\infty} D(s)^{-1} M(s)^{-1}\left|V_{0}(s)\right|^{\alpha-2}\left(V_{0}(s)\right)^{2} \overline{V_{1}(s)} d s \\
& \quad=-\frac{1}{2}\left(\frac{t^{1-\theta}}{2^{\theta}(\theta-1)}\right)^{2}\left|\mathcal{F} \phi_{+}\right|^{2 \alpha} \mathcal{F} \phi_{+} .
\end{aligned}
$$

Similarly, since $w_{n}$ is defined by

$$
w_{n}(t)=\sum_{i_{1}+\cdots+i_{\alpha+1}=n-1} H\left(w_{i_{1}}, \ldots, w_{i_{\alpha+1}}\right),
$$

the above argument implies that we should define

$$
V_{n}(t)=\sum_{i_{1}+\cdots+i_{\alpha+1}=n-1} \tilde{H}\left(V_{i_{1}}, \ldots, V_{i_{\alpha+1}}\right),
$$

where

$$
\widetilde{H}\left(u_{1}, \ldots, u_{\alpha+1}\right)=i M(t) D(t) \int_{t}^{\infty} D(s)^{-1} M(s)^{-1} \prod_{l=1}^{\alpha / 2+1} u_{l}(s) \prod_{l=\alpha / 2+2}^{\alpha+1} \overline{u_{l}(s)} d s .
$$

The function $V_{n}$ defined by (1.22) is obtained by this inductive definition.

Proposition 5.1. Let $V_{n}$ be defined by (1.22). Let $\widetilde{H}$ be defined by (5.1). Then, $V_{n}$ has the following alternative definition:

$$
V_{0}(t)=M(t) D(t) \mathcal{F} \phi_{+}, \quad V_{n}(t)=\sum_{i_{1}+\cdots+i_{\alpha+1}=n-1} \tilde{H}\left(V_{i_{1}}, \ldots, V_{i_{\alpha+1}}\right) .
$$

Proof. We show that $V_{n}$ defined by this inductive definition is equal to (1.22). Putting $v_{n}=D^{-1} M^{-1} V_{n}$, we have the definition of $v_{n}$ :

$$
v_{0}(t)=\mathcal{F} \phi_{+}, \quad v_{n}(t)=\sum_{i_{1}+\cdots+i_{\alpha+1}=n-1} i \int_{t}^{\infty}|2 s|^{-\theta} \prod_{k=1}^{\alpha / 2+1} v_{i_{k}} \prod_{k=\alpha / 2+2}^{\alpha+1} \overline{v_{i_{k}}} d s .
$$

We verify by induction that $v_{n}$ takes the following form:

$$
v_{n}(t)=J(n) \frac{t^{n(1-\theta)}}{2^{n \theta}(\theta-1)^{n}}\left|\mathcal{F} \phi_{+}\right|^{\alpha n} \mathcal{F} \phi_{+},
$$


where $J(n)$ is a sequence of complex numbers defined by $J(0)=1$ and

$$
J(n)=\frac{i}{n} \sum_{i_{1}+\cdots+i_{\alpha+1}=n-1} \prod_{k=1}^{\alpha / 2+1} J\left(i_{k}\right) \prod_{k=\alpha / 2+2}^{\alpha+1} \overline{J\left(i_{k}\right)} .
$$

If $J(n)=(i)^{n} / n$ !, then $V_{n}=M D v_{n}$ is identical to (1.22). Hence, we prove this.

We set the formal Taylor series $g(x)=\sum_{n=0}^{\infty} J(n) x^{n}$. If $x \in \mathbb{R}$, then this function $g$ satisfies

$$
\begin{aligned}
|g|^{\alpha} g & =\sum_{n=0}^{\infty}\left(\sum_{i_{1}+\cdots+i_{\alpha+1}=n} \prod_{k=1}^{\alpha / 2+1} J\left(i_{k}\right) \prod_{k=\alpha / 2+2}^{\alpha+1} \overline{J\left(i_{k}\right)}\right) x^{n} \\
& =\sum_{n=0}^{\infty}(-i)(n+1) J(n+1) x^{n}=-i g^{\prime},
\end{aligned}
$$

that is, $g^{\prime}=i|g|^{\alpha} g$. We consider this equation for $x \in \mathbb{R}$. Multiplying $\bar{g}$ and taking the real part, we first deduce that $|g|$ is constant. Because $g(0)=1$, the equation for $g$ becomes $g^{\prime}=i g$. Therefore, $g(x)=e^{i x}$. Then, we obtain $J(n)=(i)^{n} / n !$.

On the other hand, if $\alpha$ is not an even integer, then the definition of $V_{n}$ for $n \geq 2$ becomes difficult. Let $w_{n}$ be defined by (1.8). Since $w_{0}$ does not depend on $\alpha$, the above argument gives $V_{0}(t)=M(t) D(t) \mathcal{F} \phi_{+}$. Let us proceed to the approximation of $w_{1}$. Since

$$
w_{1}(t)=G\left(\left|w_{0}\right|^{\beta}, \ldots,\left|w_{0}\right|^{\beta}, w_{0}\right)=i \int_{t}^{\infty} U(t-s)\left|w_{0}(s)\right|^{\alpha} w_{0}(s) d s,
$$

the previous formal replace argument suggests that

$$
\begin{aligned}
V_{1} & =i M(t) D(t) \int_{t}^{\infty} D(s)^{-1} M(s)^{-1}\left|V_{0}(s)\right|^{\alpha} V_{0}(s) d s \\
& =i \frac{t^{1-\theta}}{2^{\theta}(\theta-1)} M(t) D(t)\left|\mathcal{F} \phi_{+}\right|^{\alpha} \mathcal{F} \phi_{+} .
\end{aligned}
$$

This is a natural extension of the previous even-number case. However, it is difficult to define $V_{2}$. The function $w_{2}$ is written as

$$
\begin{aligned}
w_{2}(t)= & \sum_{i_{1}+\cdots+i_{\lceil\alpha\rceil+1}=1} G\left(\widetilde{w}_{i_{1}}, \ldots, \widetilde{w}_{i_{\lceil\alpha\rceil}}, w_{i_{\lceil\alpha\rceil+1}}\right) \\
= & \lceil\alpha\rceil i \int_{t}^{\infty} U(t-s)\left(\left|w_{1}(s)+w_{0}(s)\right|^{\beta}-\left|w_{0}(s)\right|^{\beta}\right)\left|w_{0}(s)\right|^{\alpha-\beta} w_{0}(s) d s \\
& +i \int_{t}^{\infty} U(t-s)\left|w_{0}\right|^{\alpha} w_{1}(s) d s .
\end{aligned}
$$

Therefore, it will be approximated by

$$
\begin{aligned}
& i\lceil\alpha\rceil M(t) D(t) \int_{t}^{\infty} D(s)^{-1} M(s)^{-1}\left(\sigma_{1}(s)\left|V_{1}(s)\right|^{\beta}\right)\left|V_{0}(s)\right|^{\alpha-\beta} V_{0}(s) d s \\
& \quad+i M(t) D(t) \int_{t}^{\infty} D(s)^{-1} M(s)^{-1}\left|V_{0}\right|^{\alpha} V_{1}(s) d s
\end{aligned}
$$


where $\sigma_{1}(t, x):=\operatorname{sign}\left(\left|w_{1}(t, x)+w_{0}(t, x)\right|^{\beta}-\left|w_{0}(t, x)\right|^{\beta}\right)$. This is because

$$
|| w_{1}(s)+\left.w_{0}(s)\right|^{\beta}-\left|w_{0}(s)\right|^{\beta}-\sigma_{1}(s)\left|V_{1}(s)\right|^{\beta}|\leq| w_{1}(s)-\left.V_{1}(s)\right|^{\beta} .
$$

Note that

$$
|| w_{1}(s)+\left.w_{0}(s)\right|^{\beta}-\left|w_{0}(s)\right|^{\beta}-\left|V_{1}(s)\right|^{\beta}|\leq| w_{1}(s)-\left.V_{1}(s)\right|^{\beta}
$$

does not hold. Then, the treatment of $\sigma_{1}(t)$ prevents our defining $V_{2}$. However, using a completely different method, we can obtain $V_{2}$ only in the case where $\alpha$ is an even number. In fact, we see that if $\alpha$ is an even number, then $V_{2}$ defined by (1.22) approximates not only the function $w_{2}$ defined by (1.21) but also $w_{2}$ defined by (1.8) (see Proposition 6.2). This means that $w_{2}$ defined by (1.8) is approximated only by the second term of (5.3) and so that the first term of (5.3) is small.

\section{The proofs of Theorems 3 and 4}

Finally, we prove Theorem 3 and complete the proof of Theorem 4. We approximate $w_{n}$ by $V_{n}$.

\subsection{The proof of Theorem 3}

Proposition 6.1. Let $N \geq 1$ and $\alpha \in\left(\gamma(N), \alpha_{0}(N)\right)$. Put $\theta=\alpha N / 2, T \gg 1$, and $I=$ $[T, \infty)$. Let $V_{0}=M(t) D(t) \mathcal{F} \phi_{+}$and $V_{1}$ be given in Theorem 2 . Let $\rho$ satisfy $1 \leq \rho<$ $(\alpha+1) \wedge 2$. Then, it holds for all $r \in[2, \infty)$ with $\delta(r) \leq \rho(r \in[2, \infty]$ if $\rho>N / 2)$ that

$$
\begin{gathered}
\left\|V_{0}\right\|_{Y^{r}(T)}=C, \quad\left\|V_{0}\right\|_{L^{(2 / \delta(r))}\left(I ; L^{r}\right)}=C T^{-\delta(r) / 2}, \\
\left\|u-V_{0}\right\|_{Y^{r}(T)}=o(1), \quad\left\|u-V_{0}\right\|_{L^{(2 / \delta(r))}\left(I ; L^{r}\right)}=o\left(T^{-\delta(r) / 2}\right) .
\end{gathered}
$$

Let $r \in[2, \infty]$ satisfy $\delta(r)<(\theta-1) \wedge 1$ and $\left(q_{0}, r_{0}\right)$ be an admissible pair. Then, it holds that

$$
\begin{gathered}
\left\|V_{1}\right\|_{Y^{r}(T)}=C T^{1-\theta}, \quad\left\|V_{1}\right\|_{L^{q_{0}\left(I ; L^{\left.r_{0}\right)}\right.}}=C T^{1-\theta-\delta(r) / 2}, \\
\left\|w_{1}-V_{1}\right\|_{Y^{r}(T)}=o\left(T^{1-\theta+\delta(r)}\right), \quad\left\|w_{1}-V_{1}\right\|_{L^{q_{0}\left(I ; L^{r_{0}}\right)}}=o\left(T^{1-\theta}\right)
\end{gathered}
$$

as long as $r$ and $r_{0}$ satisfy $\delta((\alpha+1) r) \leq \rho$ and $\delta\left((\alpha+1) r_{0}\right) \leq \rho$, respectively.

Proof. It follows by the definition of $V_{0}$ that

$$
\begin{gathered}
\left\|V_{0}\right\|_{Y^{r}(T)}=2^{-\delta(r)}\left\|\mathcal{F} \phi_{+}\right\|_{L^{r}}<\infty \\
\left\|w_{0}-V_{0}\right\|_{Y^{r}(T)}=\sup _{t \geq T}\left\|\left(\mathcal{F}(M-1) \mathcal{F}^{-1}\right) \mathcal{F} \phi_{+}\right\|_{L^{r}}=o(1)
\end{gathered}
$$

for all $r \in[2, \infty)$ with $\delta(r) \leq \rho(r \in[2, \infty]$ if $\rho>N / 2)$. Now, we turn to the estimate of $V_{1}$. Assume that $\alpha N / 2(\alpha+1) \leq \rho$. An elementary calculation provides

$$
\left\|V_{1}(t)\right\|_{L^{r}}=\frac{t^{1-\theta}}{2^{\theta}(\theta-1)}(2 t)^{-\delta(r)}\left\|\left|\mathcal{F} \phi_{+}\right|^{\alpha} \mathcal{F} \phi_{+}\right\|_{L^{r}} .
$$

The $L^{r}$ norm in the right-hand side is bounded for all $r \in[2, \infty]$ with $\delta((\alpha+1) r) \leq \rho$; there exists a non-zero constant depending on $r$ such that

$$
\left\|V_{1}\right\|_{Y^{r}(T)}=C_{0} T^{1-\theta}
$$


if $\mathcal{F} \phi_{+}$is not identically zero. Similarly, one sees that

$$
\left\|V_{1}\right\|_{L^{q_{0}\left(I ; L^{r} 0\right)}}=C T^{1-\theta-\delta\left(r_{0}\right) / 2} .
$$

Therefore, it follows from (2.1), (4.4), (6.1), and (6.2) that

$$
\begin{aligned}
\| w_{1} & -V_{1} \|_{Y^{r}(T)} \\
\leq & \left\|G\left(\widetilde{w}_{0}, \ldots, \widetilde{w}_{0}, w_{0}-V_{0}\right)\right\|_{Y^{r}(T)} \\
& +\sum_{\substack{1 \leq l \leq\lceil\alpha\rceil \\
i_{1}=\cdots=i\lceil\alpha\rceil+1}}\left\|G\left(\widetilde{w}_{i_{1}}, \ldots, \widetilde{w}_{i_{l-1}}, \widetilde{w}_{i_{l}}-\left|V_{0}\right|^{\beta},\left|V_{0}\right|^{\beta}, \ldots,\left|V_{0}\right|^{\beta}, V_{0}\right)\right\|_{Y^{r}(T)} \\
& +\left\|E_{1}\right\|_{Y^{r}(T)}=o\left(T^{1-\theta+\delta(r)}\right)
\end{aligned}
$$

for $r$ with $\delta(r)<1 \wedge(\theta-1)$, where we apply Lemma 2.5 and the same argument as in the proofs of (4.5) and (4.6). This inequality holds if $N \alpha / 2(\alpha+1) \leq \rho$ and $\delta((\alpha+1) r) \leq \rho$. We note that $N \alpha / 2(\alpha+1)=\delta((\alpha+1) 2) \leq \delta((\alpha+1) r)$ if $r \geq 2$, and so that the second condition is stronger. Similarly, (2.3) provides

$$
\left\|w_{1}-V_{1}\right\|_{L^{q_{0}\left(I ; L^{r_{0}}\right)}}=o\left(T^{1-\theta}\right),
$$

where $\left(q_{0}, r_{0}\right)$ is an admissible pair such that $\delta\left((\alpha+1) r_{0}\right) \leq \rho$.

Proof of Theorem 3. This theorem immediately follows from Proposition 6.1 and the second part of Proposition 4.1 by the use of triangle inequality

$$
\left\|u-w_{0}-V_{1}\right\| \leq\left\|u-w_{0}-w_{1}\right\|+\left\|w_{1}-V_{1}\right\| .
$$

If $\alpha$ is an even number the we can obtain the asymptotic form of $w_{2}$.

Proposition 6.2. Let $N=1,2$, 3. Let $\alpha \in\left(\gamma(N), \alpha_{0}(N)\right)$ be an even number. Put $\theta=$ $\alpha N / 2$. Let $\rho \in[1,(\alpha+1) \wedge 1)$ satisfy $\rho>N / 2$. Let $T \gg 1$ and $I=[T, \infty)$. Let $V_{2}$ be a function defined by (1.22) with $n=2$. Then,

$$
\begin{gathered}
\left\|w_{2}-V_{2}\right\|_{L^{q_{0}}\left(I ; L^{\left.r_{0}\right)}\right.}=o\left(T^{2(1-\theta)}\right), \quad\left\|w_{2}(t)\right\|_{L^{2}} \asymp t^{2(1-\theta)}, \\
\left\|u(t)-w_{0}(t)-w_{1}(t)\right\|_{L^{2}} \asymp t^{2(1-\theta)} .
\end{gathered}
$$

Proof. Let $w_{n}$ and $\widetilde{w}_{n}$ be functions defined by (1.8) and (1.21), respectively. One sees that $w_{0}=\widetilde{w}_{0}$ and $w_{1}=\widetilde{w}_{1}$. Then, from Theorem 4 (we prove this in Section 6.2), we deduce for all $r \in[2, \infty]$ that

$$
\begin{gathered}
\left\|u-w_{0}-w_{1}-\widetilde{w}_{2}\right\|_{Y^{r}(T)} \leq\left\|u-w_{0}-w_{1}-\widetilde{w}_{2}\right\|_{\Sigma^{\rho}(T)} \leq C T^{3(1-\theta)}, \\
\left\|\tilde{w}_{2}-V_{2}\right\|_{Y^{r}(T)} \leq\left\|\widetilde{w}_{2}-V_{2}\right\|_{\Sigma^{\rho}(T)}=o\left(T^{2(1-\theta)}\right) .
\end{gathered}
$$

By the definition of $Y^{r}(T)$, it means that

$$
\begin{gathered}
\left\|u-w_{0}-w_{1}-\widetilde{w}_{2}\right\|_{L^{q_{0}}\left(I ; L^{\left.r_{0}\right)}\right.} \leq C T^{3(1-\theta)-\delta\left(r_{0}\right) / 2}, \\
\left\|\widetilde{w}_{2}-V_{2}\right\|_{L^{q_{0}}\left(I ; L^{r_{0}}\right)}=o\left(T^{2(1-\theta)-\delta\left(r_{0}\right) / 2}\right) .
\end{gathered}
$$


Moreover, from Theorem 2, we also deduce that $\left\|u-w_{0}-w_{1}-w_{2}\right\|_{L^{q_{0}\left(I ; L^{r}\right)}}=$ $o\left(T^{2(1-\theta)}\right)$. Therefore, we conclude that

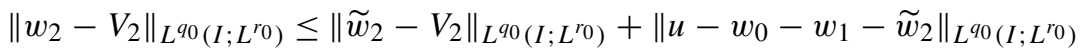

$$
\begin{aligned}
& +\left\|u-w_{0}-w_{1}-w_{2}\right\|_{L^{q_{0}\left(I ; L^{\left.r_{0}\right)}\right.}}=o\left(T^{2(1-\theta)}\right) .
\end{aligned}
$$

The lower $L^{2}$ estimates hold from this and $\left\|V_{2}(t)\right\|_{L^{2}}=C t^{2(1-\theta)}$.

\subsection{The completion of the proof of Theorem 4}

Completion of the proof of Theorem 4. By the definition of $V_{n}(1.22)$, we have

$$
\left\|V_{n}\right\|_{\Sigma^{\rho}(T)}=\frac{T^{n(1-\theta)}}{n ! 2^{n \theta}(\theta-1)^{n}}\left\|\left|\mathcal{F} \phi_{+}\right|^{\alpha n} \mathcal{F} \phi_{+}\right\|_{H^{\rho}} .
$$

Since the $H^{\rho}$ norm of $\left|\mathcal{F} \phi_{+}\right|^{\alpha n+1} \mathcal{F} \phi_{+}$is bounded by $C\left\|\mathcal{F} \phi_{+} ; H^{\rho}\right\|^{\alpha n+1}$, they are positive finite. Therefore, there exists a constant $C_{0}$ such that $\left\|V_{n} ; \Sigma^{\rho}(T)\right\|=C_{0} T^{n(1-\theta)}$. This constant is positive unless $\phi_{+}$is identically zero. Similarly, we have $\left\|V_{n} ; Y^{r}(T)\right\|=$ $C_{0} T^{n(1-\theta)}$.

Let us proceed to the proof of (1.26). We prove it by induction. Because $w_{0}-V_{0}=$ $M D F(M-1) \phi_{+}$, one sees that if $\mathcal{F} \phi_{+} \in H^{\rho}$, then

$$
\left\|w_{0}-V_{0}\right\|_{H^{\rho}}=\left\|(M(t)-1)\left(1+|x|^{2}\right)^{\rho / 2} \phi_{+}\right\|_{L^{2}} \rightarrow 0
$$

as $t \rightarrow 0$. Therefore, we have $\left\|w_{0}-V_{0}\right\|_{\Sigma^{\rho}(T)} \rightarrow 0$ as $T \rightarrow \infty$. By the multilinearity of $H$, we have

$$
\begin{aligned}
w_{n}-V_{n}= & \sum_{i_{1}+\cdots+i_{\alpha+1}=n-1} \sum_{l=1}^{\alpha+1} H\left(w_{i_{1}}, \ldots, w_{i_{l-1}}, w_{i_{l}}-V_{i_{l}}, V_{i_{l+1}}, \ldots, V_{i_{\alpha+1}}\right) \\
& +\left(\sum_{i_{1}+\cdots+i_{\alpha+1}=n-1} H\left(V_{i_{1}}, \ldots, V_{i_{\alpha+1}}\right)-V_{n}\right) .
\end{aligned}
$$

We denote the second term $\sum H\left(V_{i_{1}}, \ldots, V_{i_{\alpha+1}}\right)-V_{n}$ by $R_{n}$. Since $\left\|w_{n} ; \Sigma^{\rho}(T)\right\|$, $\left\|V_{n} ; \Sigma^{\rho}(T)\right\|=O\left(T^{n(1-\theta)}\right)$, if we assume that (1.26) holds for $n \leq n_{0}-1$, then we deduce from Lemma 2.3 that

$$
\begin{aligned}
\left\|w_{n}-V_{n}\right\|_{\Sigma^{\rho}(T)} \leq & \sum_{i_{1}+\cdots+i_{\alpha+1}=n-1} \sum_{l=1}^{\alpha+1} C_{1} T^{1-\theta}\left(\prod_{k=1}^{l-1}\left\|w_{i_{k}}\right\|_{\Sigma^{\rho}(T)}\right)\left\|w_{i_{l}}-V_{i_{l}}\right\|_{\Sigma^{\rho}(T)} \\
& \times\left(\prod_{k=l+1}^{\alpha+1}\left\|V_{i_{k}}\right\|_{\Sigma^{\rho}(T)}\right)+\left\|R_{n}\right\|_{\Sigma^{\rho}(T)} \\
= & \left\|R_{n}\right\|_{\Sigma^{\rho}(T)}+o\left(T^{n(1-\theta)}\right) .
\end{aligned}
$$


Hence it suffices to show that $\left\|R_{n}\right\|_{\Sigma^{\rho}(T)}=o\left(T^{n(1-\theta)}\right)$. We deduce from Proposition 5.1 that

$$
\begin{aligned}
R_{n} & =\sum_{i_{1}+\cdots+i_{\alpha+1}=n-1} H\left(V_{i_{1}}, \ldots, V_{i_{\alpha+1}}\right)-V_{n} \\
& =\sum_{i_{1}+\cdots+i_{\alpha+1}=n-1}(H-\widetilde{H})\left(V_{i_{1}}, \ldots, V_{i_{\alpha+1}}\right) \\
& =\sum_{i_{1}+\cdots+i_{\alpha+1}=n-1} i M(t) D(t) \int_{t}^{\infty} \mathcal{U}(t, s) D(s)^{-1} M(s)^{-1} \prod_{k=1}^{\alpha / 2+1} V_{i_{k}} \prod_{k=\alpha / 2+2}^{\alpha+1} \overline{V_{i_{k}}} d s,
\end{aligned}
$$

where $\mathcal{U}(t, s)=\mathcal{F} M(t) M(s)^{-1} \mathcal{F}^{-1}-1$. Using the explicit definition of $V_{n}$ and the definition of $J(n)$ ((1.22) and (5.2), respectively), we obtain

$$
\begin{aligned}
R_{n}= & i M(t) D(t) \int_{t}^{\infty} \mathcal{U}(t, s)|2 s|^{-\theta}\left(\frac{s^{1-\theta}}{2^{\theta}(\theta-1)}\right)^{n-1}\left|\mathcal{F} \phi_{+}\right|^{\alpha n} \mathcal{F} \phi_{+} d s \\
& \times \sum_{i_{1}+\cdots+i_{\alpha+1}=n-1} \prod_{k=1}^{\alpha / 2+1} J\left(i_{k}\right) \prod_{k=\alpha / 2+2}^{\alpha+1} \overline{J\left(i_{k}\right)} \\
= & \frac{1}{2^{n \theta}(\theta-1)^{n-1}}\left(i M(t) D(t) \int_{t}^{\infty} \mathcal{U}(t, s) s^{n(1-\theta)-1}\left|\mathcal{F} \phi_{+}\right|^{\alpha n} \mathcal{F} \phi_{+} d s\right) \frac{n}{i} J(n) \\
= & \frac{(i)^{n-1}}{(n-1) !} \times \frac{1}{2^{n \theta}(\theta-1)^{n-1}} E_{n}(t) .
\end{aligned}
$$

Then, Proposition 2.6 provides the desired estimate and completes the proof of (1.26). We finally obtain the lower bound estimates:

$$
\left\|w_{n}\right\|_{\Sigma^{\rho}(T)} \geq\left\|V_{n}\right\|_{\Sigma^{\rho}(T)}-\left\|w_{n}-V_{n}\right\|_{\Sigma^{\rho}(T)} \geq c T^{n(1-\theta)}
$$

and so

$$
\left\|u-\sum_{k=0}^{n} w_{k}\right\|_{\Sigma^{\rho}(T)} \geq\left\|w_{n+1}\right\|_{\Sigma^{\rho}(T)}-\left\|u-\sum_{k=0}^{n+1} w_{k}\right\|_{\Sigma^{\rho}(T)} \geq c T^{(n+1)(1-\theta)} .
$$

The lower bound estimates of $Y^{r}(T)$ also follow from $\left\|V_{n} ; Y^{r}(T)\right\|=C_{0} T^{n(1-\theta)}$ and the embedding $\Sigma^{\rho}(T) \hookrightarrow Y^{r}(T)$.

Acknowledgements. The author expresses his deep gratitude to Professor Yoshio Tsutsumi for his kind guidance and constant encouragement. The author also thanks Doctor Hajime Kaneko who pointed out that $I(n, k)$ defined by (3.5) is the generalized Catalan number, which is a great help for the proof of convergence results in Theorems 3.4 and 3.6.

\section{REFERENCES}

[1] I. Bejenaru and T. Tao. Sharp well-posedness and ill-posedness results for a quadratic non-linear Schrödinger equation. J. Funct. Anal. 233 (2006), 228-259.

[2] T. Cazenave and F. B. Weissler. Rapidly decaying solutions of the nonlinear Schrödinger equation. Commun. Math. Phys. 147 (1992), 75-100. 
[3] G. C. Dong and S. Li. On the initial value problem for a nonlinear Scrödinger equation. J. Differential Equations 42 (1981), 353-365.

[4] J. Ginibre, T. Ozawa and G. Velo. On the existence of the wave operator for a class of nonlinear Schrödinger equations. Ann. Inst. H. Poincaré Phys. Théor. 60 (1994), 211-239.

[5] J. Ginibre and G. Velo. On a class of nonlinear Schrödinger equations. J. Funct. Anal. 32 (1979) 1-71.

[6] J. Ginibre and G. Velo. Scattering theory in the energy space for a class of nonlinear Schrödinger equations. J. Math. Pures Appl. 64 (1985), 363-401.

[7] N. Hayashi and P. I. Naumkin. Asymptotics for large time of solutions to the nonlinear Schrödinger and Hartree equation. Am. J. Math. 120 (1998), 369-389.

[8] N. Hayashi and T. Ozawa. Scattering theory in the weighted $L^{2}\left(\mathbb{R}^{n}\right)$ spaces for some Schrödinger equations. Ann. Inst. H. Poincaré Phys. Théor. 48 (1988), 17-37.

[9] P. Hilton and J. Pedersen. Catalan numbers, their generalization, and their uses. Math. Intelligencer 13(2) (1991), 64-75.

[10] T. Kato. On nonlinear Schrödinger equations. Ann. Inst. H. Poincaré Phys. Théor. 46 (1987), 113-129.

[11] N. Kita. Sharp $L^{r}$ asymptotics of the small solutions to the nonlinear Schrödinger equations. Nonlinear Anal. 52 (2003), 1365-1377.

[12] N. Kita and T. Ozawa. Sharp asymptotic behavior of solutions to nonlinear Schrödinger equations with repulsive interactions. Comm. Contemp. Math. 7 (2005), 167-176.

[13] N. Kita and T. Wada. Sharp asymptotic behavior of solutions to nonlinear Schrödinger equations in one space dimension. Funkcial. Ekvac. 45 (2002), 53-69.

[14] S. Masaki. Semi-classical analysis for Hartree equations in some supercritical cases. Ann. Henri Poincaré 8 (2007), 1037-1069.

[15] K. Nakanishi and T. Ozawa. Remarks on scattering for nonlinear Schrödinger equations. NoDEA 9 (2002), 45-68.

[16] W. A. Strauss. Nonlinear scattering at low energy. J. Funct. Anal. 41 (1981), 110-133.

[17] Y. Tsutsumi. Scattering problem for nonlinear Schrödinger equations. Ann. Inst. H. Poincaré Phys. Théor. 43 (1985), 321-347.

[18] T. Wada. Asymptotic expansion of the solution to the nonlinear Schrödinger equation with nonlocal interaction. J. Funct. Anal. 180 (2001), 11-30.

\author{
Satoshi Masaki \\ Department of Mathematics \\ Graduate School of Science, Kyoto University \\ Sakyo-ku Kyoto 606-8502, Japan \\ (E-mail: machack@math.kyoto-u.ac.jp)
}

